\title{
STEADY STATES AND THEIR STABILITY OF HOMOGENEOUS, RIGID, EXTENDED NEMATIC POLYMERS UNDER IMPOSED MAGNETIC FIELDS*
}

\author{
GUANGHUA JI ${ }^{\dagger}$, QI WANG ${ }^{\ddagger}$, PINGWEN ZHANG ${ }^{\S}$, \\ HONGYUN WANG $\llbracket$, AND HONG ZHOUll
}

\begin{abstract}
We study the steady state phase behavior of homogeneous, rigid, extended (polar) nematic polymers or nematic dispersions under imposed magnetic (or electric fields), in which the intermolecular dipole-dipole and excluded volume interaction as well as external field contribution are accounted for. We completely characterize the phase diagram for polar nematics with and without permanent magnetic moments (or dipoles). For nematics without magnetic moments, the steady state is either purely nematic or polar depending on the strength of the excluded volume and the dipole-dipole interaction, in which the nonzero polarity vector (the first moment vector) is coaxial with the second moment tensor; thereby the steady state pdf is determined essentially by up to three scalar order parameters and a rotational group of $\mathrm{SO}(2)$ transverse to the imposed field direction. For nematics with permanent magnetic moments (or dipoles), the steady states are polar and the polarity vector is parallel to the external field direction when a necessary condition for parameters is met. When the condition is violated, only stable steady states have their polarity vector parallel to the external field direction and there are thermodynamically unstable nonparallel states. The stability of the steady states is inferred from the minimum of the free energy density.
\end{abstract}

Key words. nematic liquid crystal, kinetic theory, polymers, magnetic field, electric field, bifurcation, steady state, phase transition, polarity, stability

AMS subject classifications. 45K05, 70K50, 82B26, 82B27

\section{Introduction}

Kinetic theory is a useful tool in modeling soft matter and complex fluids [2, $4,8,24,9]$. In the past, it has been perceived as colossal and complicated-hardly accessible to theoretical analysis. Given the rising interest in kinetic theory in the mathematics community these days, various attempts have been made to analyze the properties of the partial differential equations in the kinetic theories and obtain their solutions semianalytically and numerically $[23,11,12,13,14,15,16,17,18,5$, $6,7,10,23,34,35,26,40,38,20]$. A recent review of the state of the art in the mathematical and numerical analysis of multi-scale models of complex fluids is given by Li and Zhang [25].

${ }^{*}$ Received: April 29, 2007; accepted (in revised version): September 24, 2007. Communicated by Shi Jin.

Effort sponsored by the Air Force Office of Scientific Research, Air Force Materials Command, USAF, under grant numbers F49550-05-1-0025 and F1ATA06313G003, and by the National Science Foundation through grants DMS-0605029 and DMS-0626180 which are gratefully acknowledged. P. Zhang is partially supported by the special funds for Major State Research Projects 2005CB321704 and National Science Foundation of China for Distinguished Young Scholars 10225103 and 20490222.

${ }^{\dagger}$ LMAM and School of Mathematical Sciences, Peking University, Beijing 100871, P.R. China, and School of Mathematical Sciences, Beijing Normal University, Beijing 100875, P.R. China (ghji@bnu.edu.cn).

$\ddagger$ Department of Mathematics, Florida state University, Tallahassee, FL 32306 and School of Mathematics, Nankai University, Tianjin 300071, P.R. China (wang@math.fsu.edu).

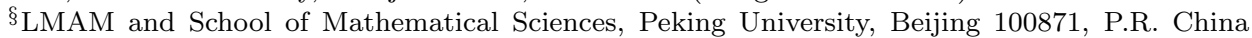
(pzhang@pku.edu.cn).

『Department of Applied Mathematics and Statistics, University of California, Santa Cruz, CA 95064.

" Department of Applied Mathematics, Naval Postgraduate School, Monterey, CA 93943 (hzhou@nps.edu). 
In a series of papers $[38,20,41,42]$, we have given a systematic study of the exact solution of the Smoluchowski equation with the Maier-Saupe excluded volume potential $[29,30,31]$ and (or) the dipole-dipole interaction potential in implicit form using a reduced order or projection method for the Doi-Hess kinetic theory [9]. In [38], we studied the phase behavior of rigid nematics dominated by either the excluded volume or the dipole-dipole interaction coupled with an imposed external field. Therein, we identified the order reduction strategy that we have adopted in all the subsequent studies in the series. In [41, 42], we proved a series of theoretical results establishing the conditions for bifurcations of steady states and the asymptotic behavior of steady states near the bifurcation point in phase space in models with both dipole-dipole and excluded volume interaction. In [20], we proved additional theoretical results for steady states of the Smoluchowski equation and obtained the complete bifurcation diagram for polar nematics absent of external fields. In the above studies, we applied the reduced order method to various limiting cases of dilute or concentrated, nematics or extended (polar) nematics, with or without the external field. By extended nematics we mean here the nematics that exhibit instantaneous dipoles or magnetic moments; therefore, the dipole-dipole interaction must be accounted for. We have established rigorous mathematical theories for the existence of certain types of solutions and their stability [38, 20,41, 42], as well as detailed phase bifurcation diagrams [38, 20]. One of the examples of extended nematics or polar nematics is the nematic liquid crystal polymer consisting of bowlike molecules, which were studied experimentally and numerically in the past [3, 21, 27, 28, 39].

In this paper, we extend the previous studies on homogeneous rigid extended or polar nematics by examining the steady states subject to the dipole-dipole and excluded volume interaction under imposed external fields in dilute and concentrated regimes with or without the permanent magnetic moments (or dipoles). This will give a closure to the study of the steady state solution behavior governed by the Smoluchowski equation with the potential given by the following form

$$
V=-k_{B} T(\mu \mathbf{E}+\alpha\langle\mathbf{m}\rangle) \cdot \mathbf{m}-\frac{3 N k_{B} T}{2}\langle\mathbf{m m}\rangle: \mathbf{m m}-\frac{\alpha_{0} k_{B} T}{2} \mathbf{E E}: \mathbf{m m},
$$

where $\mu$ is the strength of the permanent dipole (or magnetic moment) of the molecule, $\alpha$ is the strength of the dipole-dipole interaction potential due to the intrinsic dipole (or magnetic moment) on the molecule, $\alpha_{0}$ is the difference of the polarizability (or susceptibility) parallel and perpendicular to the molecular direction known as the anisotropy, $\mathbf{E}$ is the external magnetic (or electric) field, $N$ is a dimensionless parameter describing the strength of the Maier-Saupe excluded volume potential and quadruple-quadruple interaction, $k_{B}$ is the Boltzmann constant and $T$ is the absolute temperature $[9,1]$. In this model, we describe the orientation of the extended nematic molecule by a unit vector $\mathbf{m}$, which is in the permanent dipole (or magnetic moment) direction if $\mu \neq 0$ so that $\mu>0$, or in the direction of the averaged dipole when $\mu=0$ so that $\alpha>0$ in the latter case. We remark that all the material parameters are normalized with respect to $k_{B} T$ in this paper. So, we effectively set $k_{B} T=1$ in all our numerical calculations.

We adopt the Maier-Saupe potential as the excluded volume potential in the studies $[29,30,31]$ because it is an approximation to the integral form Onsager potential [9]. Although the Maier-Saupe and Onsager potential give the same behavior qualitatively, theoretical analysis with an Onsager potential would require a completely new set of tools which are yet to be discovered, because the Onsager potential requires a complete set of spherical harmonic modes, while the Maier-Saupe only needs 
the first few. It is this property of the Maier-Saupe potential that results in our reduced order method which has led to many in-depth theoretical results recently $[5,6,7,10,34,35,38,20,41,42]$.

The steady state solution of the Smoluchowski equation

$$
\frac{\partial}{\partial t} f=\mathcal{R} \cdot\left(D_{r} f \mathcal{R} U\right)
$$

in the Doi-Hess kinetic theory with the normalized chemical potential $U=\ln f+\frac{V}{k_{B} T}$ is given by $[19,9,33,37,35,38]$

$$
f(\mathbf{m})=\frac{1}{Z} e^{-V / k_{B} T},
$$

where $f$ is the orientational probability density function (pdf) of the ensemble of the extended nematic molecules, $Z$ denotes the normalizing constant or the partition function, $V$ is the total potential, $\mathcal{R}=\mathbf{m} \times(\partial / \partial \mathbf{m})$ is the rotational gradient operator, and $D_{r}$ is the rotational diffusivity, which is taken to be a material constant in this study.

A direct consequence from the above steady state solution is that the total torque, the total ensemble averaged torque, as well as the averaged external torque all vanish at steady states; i.e.,

$$
\langle\mathcal{R} U\rangle=\langle\mathcal{R} V\rangle=\left\langle\mathcal{R} V_{e}\right\rangle=\mathbf{0},
$$

where $V_{e}=k_{B} T\left(-\frac{\alpha_{0}}{2} \mathbf{E E}: \mathbf{m m}-\mu \mathbf{E} \cdot \mathbf{m}\right)$ is the external potential due to the external field $[41,42]$. In fact, in steady states,

$$
U=\text { const. }
$$

Then,

$$
0=\mathcal{R} U=\langle\mathcal{R} U\rangle=\langle(\mathcal{R} \ln f+\mathcal{R} V)\rangle=\langle\mathcal{R} V\rangle
$$

since

$$
\langle\mathcal{R} \ln f\rangle=\int_{\|\mathbf{m}\|=1} \mathcal{R} f d \mathbf{m}=0 .
$$

In addition, we denote the intermolecular potential by

$$
V_{i}=k_{B} T\left(-\alpha\langle\mathbf{m}\rangle \cdot \mathbf{m}-\frac{3 N}{2} \mathbf{M}: \mathbf{m m}\right),
$$

where $\mathbf{M}=\langle\mathbf{m m}\rangle$. Then,

$$
\begin{aligned}
\left\langle\mathcal{R} V_{i}\right\rangle & =k_{B} T\langle\mathbf{m} \times(-\alpha\langle\mathbf{m}\rangle-3 N \mathbf{M} \cdot \mathbf{m})\rangle \\
& =k_{B} T[-\alpha\langle\mathbf{m}\rangle \times\langle\mathbf{m}\rangle-3 N\langle\mathbf{m} \times \mathbf{M} \cdot \mathbf{m}\rangle] \\
& =-3 N k_{B} T M_{i l} M_{l j} \epsilon_{i j n}=0
\end{aligned}
$$

where $\epsilon_{i j k}$ is the permutation symbol [2]. Thus,

$$
\left\langle\mathcal{R} V_{e}\right\rangle=\left\langle\mathcal{R}\left(V-V_{i}\right)\right\rangle=0 .
$$


The steady state pdf given in (1.3) is parametrized by the material and external field parameters along with the first and second moment of $\mathbf{m}$ with respect to the pdf defined below,

$$
\langle\mathbf{m}\rangle=\int_{\|\mathbf{m}\|=1} \mathbf{m} f(\mathbf{m}) d \mathbf{m},\langle\mathbf{m m}\rangle=\int_{\|\mathbf{m}\|=1} \mathbf{m m} f(\mathbf{m}) d \mathbf{m},
$$

which consists of a total of 8 nonlinear, implicit algebraic and integral equations. In $[20,41,42]$, we studied the case where the external field is absent by developing an order-reduction method. We showed that the polarity vector (or the first moment vector) must be either zero or coaxial with the nematic order tensor (or the second moment), thereby reducing the parameterizing equation of the Boltzmann function to three equations for the scalar order parameters in the eigen-frame of the nematic order tensor. Then, a comprehensive bifurcation diagram for the equilibrium phases was obtained.

In the following, we extend the order reduction strategy to extended nematics under imposed external fields. We will present the phase diagram for the extended nematics at various strengths of the imposed external field and characterize the various phases with respect to the material and external field parameters. We present the results first for the case without the permanent dipole or magnetic moment and then discuss the case with the permanent dipole.

\section{Extended nematics without permanent dipoles}

When the permanent dipole is absent, $\mu=0$. We will establish the relation between the polarity vector, the external field and the second order nematic tensor by showing that the external field must be parallel to one of the principal axes of the nematic order tensor. The theoretical foundation developed in [20, 41, 42] then enables us to extend the order-reduction scheme to study the steady state solutions of the Smoluchowski equation.

First, we show that the external field must be one of the eigenvectors of the nematic order tensor in steady state.

THEOREM 2.1. The external field is parallel to one of the principal axes of the nematic order tensor (i.e., the second moment of the pdf).

Proof. It follows from the discussion on the ensemble averaged torque alluded to in the introduction that

$$
\begin{aligned}
-\frac{1}{k_{B} T}\langle\mathcal{R} V\rangle & =\alpha\langle\mathbf{m}\rangle \times\langle\mathbf{m}\rangle+\alpha_{0}\langle\mathbf{E} \cdot \mathbf{m m} \times \mathbf{E}\rangle+3 N\langle\mathbf{m} \times \mathbf{M} \cdot \mathbf{m}\rangle \\
& =\alpha_{0}\langle\mathbf{E} \cdot \mathbf{m m} \times \mathbf{E}\rangle+3 N\langle\mathbf{m} \times \mathbf{M} \cdot \mathbf{m}\rangle=\alpha_{0}\langle\mathbf{E} \cdot \mathbf{m m} \times \mathbf{E}\rangle=0,
\end{aligned}
$$

where $\mathbf{M}=\langle\mathbf{m m}\rangle$. Then,

$$
\|\langle\mathbf{E} \cdot \mathbf{m m} \times \mathbf{E}\rangle\|^{2}=\|\mathbf{E}\|^{2}\|\mathbf{M} \cdot \mathbf{E}\|^{2}-(\mathbf{M}: \mathbf{E E})^{2}=0 .
$$

This equality holds if and only if

$$
\mathbf{M} \cdot \mathbf{E} \| \mathbf{E} .
$$

Namely, $\mathbf{E}$ is one of the principal axes of the nematic order tensor $\mathbf{M}$.

The next theorem shows that the polarity vector is related to an eigenvector of the nematic order tensor when it is nonzero. 
THEOREM 2.2. The polarity vector is either zero or must be parallel to one of the principal axes of the nematic order tensor.

Proof. We select $x, y$, and $z$ axes such that the second moment is diagonal. That is, $\left\langle m_{i} m_{j}\right\rangle=0$ for $i \neq j$. We want to prove that $\langle\mathbf{m}\rangle$ is either zero or parallel to one of the principal axes of the second moment $\langle\mathbf{m m}\rangle$.

First we denote the principal axes of $\langle\mathbf{m m}\rangle$ as $\mathbf{e}_{1}, \mathbf{e}_{2}$ and $\mathbf{e}_{3}$ and $\mathbf{E}=E \mathbf{e}_{k}$ for $1 \leq k \leq 3$. Let $\langle\mathbf{m}\rangle=r_{1} \mathbf{e}_{1}+r_{2} \mathbf{e}_{2}+r_{3} \mathbf{e}_{3}$. Suppose at least two of $r_{1}, r_{2}$ and $r_{3}$ are non-zero (otherwise $\langle\mathbf{m}\rangle$ is already parallel to one of the principal axes of $\langle\mathbf{m m}\rangle$ ). Without loss of generality, we assume both $r_{1}>0$ and $r_{2}>0$ (we can always rename the $x, y, z$ axes to achieve this). Then the total potential is

$$
\begin{aligned}
\frac{V\left(m_{1}, m_{2}, m_{3}\right)}{k_{B} T} & =-\alpha\left(r_{1} m_{1}+r_{2} m_{2}+r_{3} m_{3}\right)-\left(c_{1} m_{1}^{2}+c_{2} m_{2}^{2}+c_{3} m_{3}^{2}\right) \\
& \equiv-\alpha\left(r_{1} m_{1}+r_{2} m_{2}+r_{3} m_{3}\right)-V_{2}\left(m_{1}, m_{2}, m_{3}\right),
\end{aligned}
$$

where $c_{j}=\frac{3 N}{2}\left\langle m_{j} m_{j}\right\rangle+\frac{\alpha_{0}}{2} E^{2} \delta_{j k}$. Notice that $V_{2}$ is an even function of $m_{1}$ and $m_{2}$. We will show that $\left\langle m_{1} m_{2}\right\rangle>0$, which contradicts the selection of the principal axes.

$$
\begin{aligned}
\left\langle m_{1} m_{2}\right\rangle= & \frac{1}{Z} \int_{\|\mathbf{m}\|=1} m_{1} m_{2} \exp \left[\alpha\left(r_{1} m_{1}+r_{2} m_{2}+r_{3} m_{3}\right)+V_{2}\left(m_{1}, m_{2}, m_{3}\right)\right] d \mathbf{m} \\
= & \frac{1}{Z} \int_{\|\mathbf{m}\|=1, m_{1}>0, m_{2}>0} m_{1} m_{2} \exp \left[\alpha r_{3} m_{3}+V_{2}\left(m_{1}, m_{2}, m_{3}\right)\right] \times \\
& \left\{\exp \left(\alpha r_{1} m_{1}+\alpha r_{2} m_{2}\right)-\exp \left(-\alpha r_{1} m_{1}+\alpha r_{2} m_{2}\right)\right. \\
& \left.-\exp \left(\alpha r_{1} m_{1}-\alpha r_{2} m_{2}\right)+\exp \left(-\alpha r_{1} m_{1}-\alpha r_{2} m_{2}\right)\right\} d \mathbf{m} \\
= & \frac{4}{Z} \int_{\|\mathbf{m}\|=1, m_{1}>0, m_{2}>0} m_{1} m_{2} \exp \left[\alpha r_{3} m_{3}+V_{2}\left(m_{1}, m_{2}, m_{3}\right)\right] \times \\
& \sinh \left(\alpha r_{1} m_{1}\right) \sinh \left(\alpha r_{2} m_{2}\right) d \mathbf{m}>0 .
\end{aligned}
$$

In $[38,20,41]$, we showed that the steady state phase is nematic for the dilute $(N=0)$ solution of rigid, extended nematics when $\alpha \leq 1$. Next we will show that this result remains true in the concentrated regime as well.

THEOREM 2.3. when $\alpha \leq 1$, the solution is nonpolar so that the external field is parallel to one of the principal axes of the nematic order tensor.

Proof. We assume $\langle\mathbf{m}\rangle=r_{1} \mathbf{e}_{1}$ and denote the total potential as

$$
V=k_{B} T\left(-\alpha m_{1} r_{1}-V_{2}\left(m_{1}, m_{2}, m_{3}\right)\right) .
$$

We note that $V_{2}\left(m_{1}, m_{2}, m_{3}\right)$ is even with respect to all its variables. We will show that when $\alpha \leq 1, r_{1}$ must be zero so the only equilibrium is non-polar. We prove it by contradiction. Suppose $r_{1}>0$ (otherwise we can change the coordinate system to achieve this).

$$
\begin{aligned}
r_{1} & =\left\langle m_{1}\right\rangle=\frac{\int_{S} m_{1} \exp \left[\alpha r_{1} m_{1}+V_{2}\left(m_{1}, m_{2}, m_{3}\right)\right] d S}{\int_{S} \exp \left[\alpha r_{1} m_{1}+V_{2}\left(m_{1}, m_{2}, m_{3}\right)\right] d S} \\
& =\frac{\int_{S \text { with } m_{1}>0} m_{1} \exp \left[V_{2}\left(m_{1}, m_{2}, m_{3}\right)\right] \sinh \left(\alpha r_{1} m_{1}\right) d S}{\int_{S \text { with } m_{1}>0} \exp \left[V_{2}\left(m_{1}, m_{2}, m_{3}\right)\right] \cosh \left(\alpha r_{1} m_{1}\right) d S},
\end{aligned}
$$


where $S=\{\mathbf{m} \mid\|\mathbf{m}\|=1\}$ denotes the unit sphere. If $\alpha \leq 0$, this expression yields $r_{1} \leq 0$, contradicting $r_{1}>0$. For $\alpha>0$ and using the fact that $\tanh (x)<x$ for $x>0$, we have

$$
\begin{aligned}
& m_{1} \exp \left[V_{2}\left(m_{1}, m_{2}, m_{3}\right)\right] \sinh \left(\alpha r_{1} m_{1}\right) \\
\leq & \exp \left[V_{2}\left(m_{1}, m_{2}, m_{3}\right)\right] \cosh \left(\alpha r_{1} m_{1}\right) \tanh \left(\alpha r_{1} m_{1}\right) \\
< & \alpha r_{1} \exp \left[V_{2}\left(m_{1}, m_{2}, m_{3}\right)\right] \cosh \left(\alpha r_{1} m_{1}\right) .
\end{aligned}
$$

Substituting this inequality into the expression for $r_{1}$ above, we obtain

$$
r_{1}<\alpha r_{1},
$$

which is a contradiction when $\alpha \leq 1$ and $r_{1}>0$. Therefore $r_{1}=0$ when $\alpha \leq 1$.

This shows that the extended nematic solution is possible only when $\alpha>1$. In order to resolve the steady states for $\alpha>1$, we denote by $\mathbf{n}, \mathbf{n}_{\perp}, \mathbf{n}^{*}$ the three orthonormal eigenvectors of the second moment tensor $\langle\mathbf{m m}\rangle$ following the order-reduction scheme in [38]. We parameterize $\mathbf{E},\langle\mathbf{m}\rangle$ and $\mathbf{m}$ with respect to the basis as follows:

$$
\begin{aligned}
& \mathbf{m}=\cos \theta \mathbf{n}+\sin \theta \cos \phi \mathbf{n}_{\perp}+\sin \theta \sin \phi \mathbf{n}^{*}, \\
& \langle\mathbf{m}\rangle=s_{1}\left[\cos \theta^{\prime} \mathbf{n}+\sin \theta^{\prime} \cos \phi^{\prime} \mathbf{n}_{\perp}+\sin \theta^{\prime} \sin \phi^{\prime} \mathbf{n}^{*}\right], \\
& \mathbf{E}=E\left[\cos \theta_{E} \mathbf{n}+\sin \theta_{E} \cos \phi_{E} \mathbf{n}_{\perp}+\sin \theta_{E} \sin \phi_{E} \mathbf{n}^{*}\right],
\end{aligned}
$$

where $\theta^{\prime}, \phi^{\prime}, \theta_{E}, \phi_{E}$ are a couple of Euler angles for the first moment vector and the external field, respectively, in the eigen-frame, $E$ is the magnitude of the external field, and $s_{1}$ is the order parameter for polarity, known as the polar order parameter [38]. We adopt the biaxial representation for the second moment tensor [36, 37]:

$$
\mathbf{M}=\langle\mathbf{m m}\rangle=s\left(\mathbf{n n}-\frac{\mathbf{I}}{3}\right)+\beta\left(\mathbf{n}_{\perp} \mathbf{n}_{\perp}-\mathbf{I} / 3\right)+\mathbf{I} / 3,
$$

where $s$ and $\beta$ are the two nematic order parameters.

The steady state probability density function is given by

$$
\begin{aligned}
f= & \frac{1}{Z} e^{h}, \\
h= & \alpha s_{1}\left(\cos \theta^{\prime} \cos \theta+\sin \theta^{\prime} \sin \theta \cos \left(\phi-\phi^{\prime}\right)\right)+\frac{\alpha_{0}}{2} E^{2}\left(\cos \theta_{E} \cos \theta+\right. \\
& \left.\sin \theta_{E} \sin \theta \cos \left(\phi-\phi_{E}\right)\right)^{2}+\frac{3 N}{2}\left[\left(s-\frac{\beta}{2}\right)\left(\cos ^{2} \theta-1 / 3\right)+\frac{\beta}{2} \cos 2 \phi \sin ^{2} \theta\right],
\end{aligned}
$$

where

$$
Z=\int_{\|\mathbf{m}\|=1} e^{h} d \mathbf{m}
$$

is the normalizing coefficient or the partition function. The order parameters are defined by

$$
s_{1}=\left\langle\cos \theta \cos \theta^{\prime}+\sin \theta \sin \theta^{\prime} \cos \left(\phi-\phi^{\prime}\right)\right\rangle, \beta=\left\langle\sin ^{2} \theta \cos 2 \phi\right\rangle, s=\left\langle P_{2}(\cos \theta)\right\rangle+\beta / 2,
$$


where $s_{1}$ is called the polar order parameter and $P_{2}(x)=\frac{3}{2} x^{2}-\frac{1}{2}$ is the second order Legendre polynomial.

According to the above established results, we can choose $\theta^{\prime}=0$, i.e., identifying the principal direction of the order tensor parallel to the polarity vector as $\mathbf{n}$. Then, $\theta_{E}=0(\mathbf{e} \| \mathbf{n})$, or $\theta_{E}=\frac{\pi}{2}$ and $\phi_{E}=0\left(\mathbf{e} \| \mathbf{n}^{\perp}\right), \frac{\pi}{2}\left(\mathbf{e} \| \mathbf{n}^{*}\right)$, where $\mathbf{e}$ is the unit vector in the direction of $\mathbf{E}$. These results effectively reduce the degrees of freedom for the polarity vector and the nematic order tensor from 8 to 3 in the eigenframe!

The stability of the steady states is inferred from the free energy density of the system. The free energy density of the extended nematic polymer system is given by $[9]$

$$
A[f]=\int_{\|\mathbf{m}\|=1}\left[k_{B} T \ln f+\frac{V_{i}}{2}+V_{e}\right] f d \mathbf{m} .
$$

From (2.10), we arrive at the free energy density at the steady state:

$$
\begin{aligned}
A[f] & =\int_{\|\mathbf{m}\|=1}\left[-k_{B} T \ln Z-\frac{V_{i}}{2}\right] f d \mathbf{m} \\
& =-k_{B} T\left[\ln Z-\frac{3 N}{4} \mathbf{M}: \mathbf{M}-\frac{\alpha}{2}\|\langle\mathbf{m}\rangle\|^{2}\right] \\
& =-k_{B} T\left[\ln Z-\frac{N}{2}\left(s^{2}-s \beta+\beta^{2}\right)-\frac{\alpha}{2} s_{1}^{2}\right]+\text { const. }
\end{aligned}
$$

To infer local stability or meta-stability, we need to calculate the second variation of the free energy density with respect to both $\mathbf{M}$ and $\langle\mathbf{m}\rangle$ [32]. For global stability, the minimum value of the free energy density (2) would be sufficient, though. In the stability analysis, the orientational variables in the free energy density should be $\langle\mathbf{m}\rangle$ and $\mathbf{M}$, where the partition function $Z$ with $h$ given by

$$
h=\alpha\langle\mathbf{m}\rangle \cdot \mathbf{m}+\frac{3 N}{2} \mathbf{M}: \mathbf{m m}+\frac{\alpha_{0}}{2} \mathbf{E E}: \mathbf{m m}
$$

is accounted for. The Hessian of the free energy density is an $8 \times 8$ matrix. The eigenvalue of the Hessian reveals the local stability properties of the equilibrium with respect to not only the order parameter perturbation but also the directors (the principal axes of the nematic order tensor as well as the polarity vector).

\section{Reduced Symmetry.}

Due to the existence of molecular dipoles, $f(\mathbf{m}, t) \neq f(-\mathbf{m}, t)$. However, if $f(\mathbf{m}, \mathbf{E}, t)$ is a solution of the Smoluchowski equation, so is $f(\mathbf{U} \cdot \mathbf{m}, \mathbf{U} \cdot \mathbf{E}, t)$ provided $\mathbf{U}$ is an orthogonal matrix. In particular, if the orthogonal transformation satisfies $\mathbf{U} \cdot \mathbf{E}=\mathbf{E}$, the solution is invariant under the orthogonal transformation. Thus, a $\mathrm{SO}(2)$ symmetry remains when $\mathbf{E} \neq \mathbf{0}$, while $S O(3)$ symmetry is valid only when $\mathbf{E}=\mathbf{0}$. The steady state solution family of the Smoluchowski equation is thus parametrized by the $\mathrm{SO}(2)$ rotational group transverse to the external field direction for any given pdf parameterized by the order parameters in an eigen-frame of the nematic order tensor.

We next nondimensionalize the permanent dipole strength and the anisotropy parameter with respect to a characteristic external field strength $E_{0}$ as follows:

$$
\chi_{0}=\alpha_{0} E_{0}^{2}, \tilde{\mu}=\mu E_{0} .
$$


When the permanent dipole is absent, we choose the characteristic field strength as $E_{0}=E$. Then, the external field is always a unit vector in dimensionless form in this case. For simplicity, we will drop the tilde on $\mu$ in the following and assume it dimensionless.

2.1. Dilute limit. We first examine the limiting case $N=0$, termed the dilute limit. When $\langle\mathbf{m}\rangle$ is parallel to $\mathbf{E}$, the polar order parameter is given by

$$
s_{1}=\langle\cos \theta\rangle,
$$

where

$$
f=\frac{1}{Z} e^{\alpha s_{1} \cos \theta+\frac{\chi_{0}}{2} \cos ^{2} \theta} .
$$

It can be simplified to

$$
s_{1}=\frac{1}{Z} \int_{-1}^{1} z e^{\alpha s_{1} z+\frac{\chi_{0}}{2} z^{2}} d z
$$

where $Z=\int_{-1}^{1} e^{\alpha s_{1} z+\frac{\chi_{0}}{2} z^{2}} d z$. Given the form of the pdf solution, the nematic order tensor is uniaxial given by

$$
\langle\mathbf{m m}\rangle=s(\mathbf{n n}-\mathbf{I} / 3)+\mathbf{I} / 3,
$$

where $s=\left\langle P_{2}(\cos \theta)\right\rangle$. We notice that the effect of the imposed field is to induce purely nematic phases $(s \neq 0)$ and facilitate the formation of the polar nematic phase $\left(s_{1} \neq 0\right)$. The left column in Figure 2.1 depicts the phase diagram in parameter space $\left(\alpha, \chi_{0}\right)$, in which the second order phase transition curve is defined by $s_{1}=\langle\cos \theta\rangle, 1-\alpha\left(\left\langle\cos ^{2} \theta\right\rangle-\right.$ $\left.s_{1}^{2}\right)=0$ in the phase space $\left(\alpha, \chi_{0}\right)$. The unique phase is purely nematic on the left of the curve and polar nematic on the right of the curve. Figure 2.1 also depicts the bifurcation diagram at fixed anisotropy $\chi_{0}=0.5$, where a pitchfork bifurcation occurs at $\alpha_{c}<3$ such that a pair of stable uniaxial polar nematic phases emerge for $\alpha>\alpha_{c}$.

In steady states, the uniaxial order parameter $s$ is positive for all $\alpha$, indicating that the material is in nematic phase so long as there is an external field $\mathbf{E}$. Figure 2.1 demonstrates that the polar order indeed enhances the nematic order [20]. The only stable solution at larger $\alpha$ is the unique, prolate, polar nematic phase since $s_{1}$ and $-s_{1}$ give the same nematic order parameter $s>0$.

When $\langle\mathbf{m}\rangle$ is perpendicular to $\mathbf{E}$, the polar order parameter is given by

$$
s_{1}=\frac{1}{Z} \int_{0}^{\pi} \int_{-1}^{1} z e^{\alpha s_{1} z-\frac{\chi_{0}}{4}\left(z^{2}+\left(z^{2}-1\right) \cos \phi\right)} d z d \phi,
$$

where $Z=\int_{-1}^{1} \int_{0}^{\pi} e^{\alpha s_{1} z-\frac{\chi_{0}}{2}\left(z^{2}+\left(z^{2}-1\right) \cos ^{2} \phi\right)} d z d \phi$. The pdf solution is given by

$$
f=\frac{1}{Z} e^{\alpha s_{1} \cos \theta+\frac{\chi_{0}}{2} \sin ^{2} \theta \cos ^{2} \phi}
$$

It is biaxial if $s_{1} \neq 0$, and the two order parameters $s$ and $\beta$ are calculated by

$$
s-\frac{\beta}{2}=\left\langle P_{2}(\cos \theta)\right\rangle, \beta=\left\langle\sin ^{2} \theta \cos 2 \phi\right\rangle .
$$

The right column in Figure 2.1 depicts the phase diagram in the parameter space $\left(\alpha, \chi_{0}\right)$, where the second order phase transition curve is defined by $s_{1}=\langle\cos \theta\rangle, 1-$ 
$\langle\mathrm{m}\rangle / \mathrm{E}$
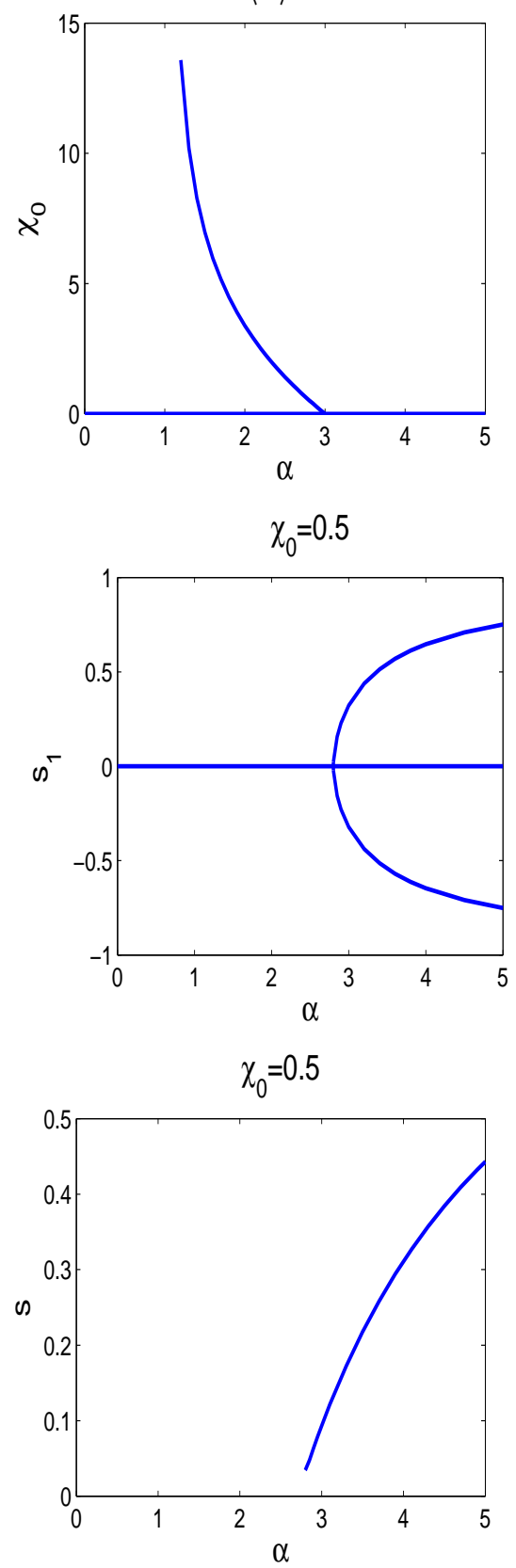

$\langle m\rangle \perp E$

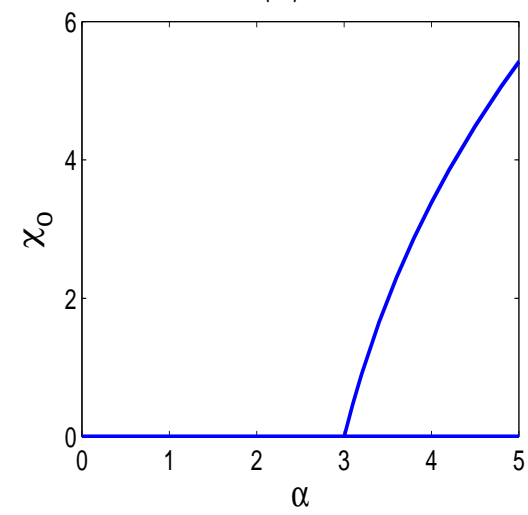

$\chi_{0}=0.5$
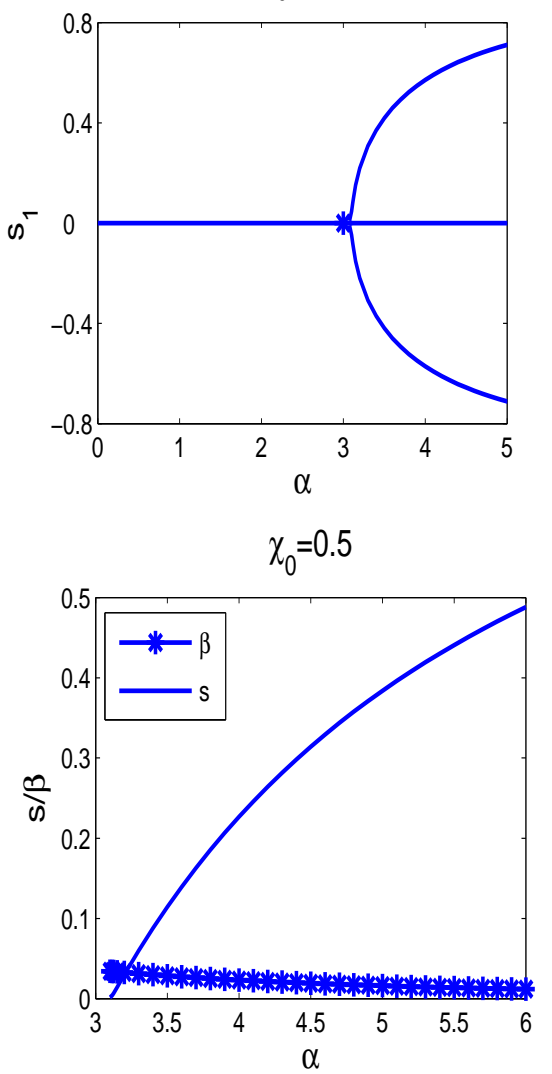

FIG. 2.1. The steady states for diluted extended nematics. The left column includes the phase diagram and order parameters when the polarity vector parallels the external field, while the right column depicts the same when the polarity vector is perpendicular to the field. $s_{1}$ is the polar order and $(s, b e t a)$ are the nematic order. When the polarity vector is parallel to the external field, the nematic phase is uniaxial (i.e., $\beta=0$ ). Notice that the nematic order parameters $(s, \beta)$ are plotted only in the range of polar nematics, and the steady state phase is nematic so long as $\chi_{0} \neq 0$. The weak biaxiality in polar nematics exists in the perpendicular situation. 
$\alpha\left(\left\langle\cos ^{2} \theta\right\rangle-s_{1}^{2}\right)=0$. The bifurcation diagram for $\chi_{0}=0.5$ in $\left(\alpha, s_{1}\right)$ is shown in Figure 2.1 , indicating that the effect of the $\mathbf{E}$ field is to delay the formation of the nematic phase to beyond $\alpha=3$. Figure 2.1 also plots the nematic order parameters $s, \beta$ of the second moment tensor in the regime of polar nematics. The formation of the biaxial phase is completely due to the emergence of the polar order parameter since the solution branch shown in Figure 2.1 is a uniaxial prolate phase $(s=0, \beta \neq 0)$ before the onset of the polar nematics. Thermodynamically, these solutions are unstable. We emphasize that the nematic order exists as long as the external field is present even when the polar order is absent (i.e., when $s=s_{1}=0, \beta \neq 0$ ). This is indeed the field-induced nematic order.

In summary, the only stable steady state under an imposed external field is uniaxial, prolate purely nematic at low $\alpha$ and prolate polar nematic at high $\alpha$ in which the director is parallel to the external field direction. The unstable purely nematic prolate phase at low $\alpha$ and the biaxial polar nematic one at high $\alpha$ exist when the external field is perpendicular to the polarity vector.

2.2. Concentrated limit: steady states with the polarity vector parallel to the external field. In this case, $\theta^{\prime}=\theta_{E}=0$. We investigate the steady states and their stability numerically using a continuation method. First we note that the governing system of equations for $\left(s_{1}, s, \beta\right)$ admits the uniaxial solution $\left(s_{1}=s=\beta=0\right)$ when the external field is absent. Along the uniaxial branch, the eigenvalue of the Hessian in the second variation of the free energy (given in Appendix) changes sign at

$$
\alpha=\frac{3}{1+2 s} .
$$

Thus, this together with the definition of $s_{1}, s$ defines the critical strength of the dipoledipole interaction potential, beyond which a polar nematic branch forms through a pitchfork bifurcation. That is,

$$
s_{1}=\langle\cos \theta\rangle, s=\left\langle P_{2}(\cos \theta)\right\rangle, \alpha=\frac{3}{1+2 s}, s>0 .
$$

This case was studied in [20] for polar nematics, where a complete phase diagram was obtained.

When a weak external field is imposed $\left(\chi_{0}<<1\right.$, a small perturbation of the situation studied in [20]), the phase diagram is surely perturbed. One prominent change is that the steady states are all nematic now showing that the external field eliminates all isotropic states and forces the steady states into nematics. Figure 2.2 depicts the phase diagram at $\chi_{0}=0.01$ and $\chi_{0}=0.1$, respectively. The pair of tricritical points connecting the first order transition curve to the second order one still exist except that they are perturbed slightly. The triple point is annihilated since the existing phases are either purely nematic and/or polar nematic. There are coexistence regions of highly aligned purely nematic and less aligned purely nematic phases and purely nematic and polar nematic phases in parameter spaces. At high concentration $N$ and/or high $\alpha$ only polar nematic phases survive. When the strength of the anisotropy $\chi_{0}$ increases further, even the tricritical points disappear, leaving an exclusive second order phase transition curve between pure nematics and polar nematics analogous to the two phase transition curves depicted in Figure 2.2 2b, 2d.

We now give a detailed account of the steady state phase behavior shown in Figure 2.2 as we vary the concentration $N$ at fixed anisotropy $\chi_{0}=0.1$ and a few 


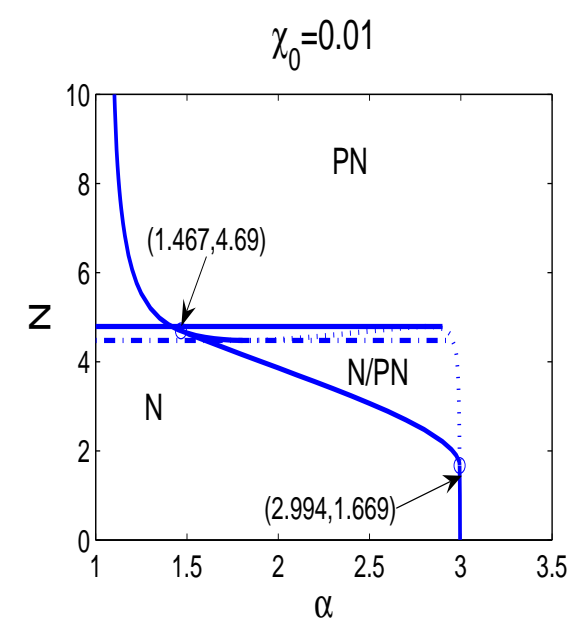

(a)

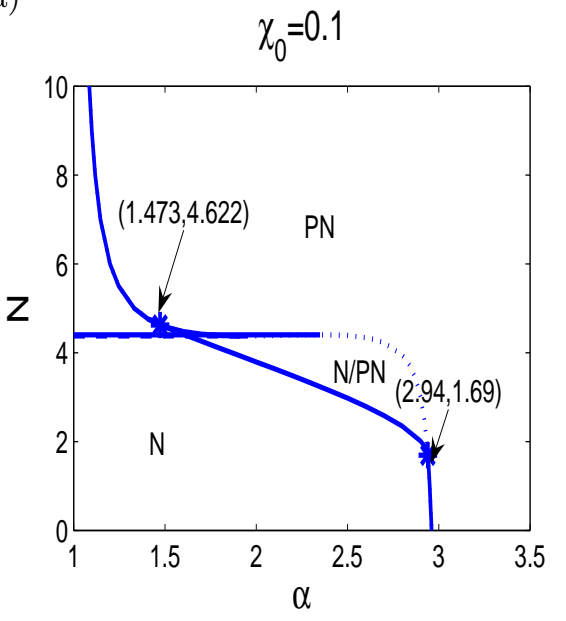

(c)

FIG. 2.2. The phase diagram in parameter space $(\alpha, N)$ when the polarity vector is parallel to the external field at $\chi_{0}=0.01$ and $\chi_{0}=0.1$, respectively. (a) and (b) depict the limit of the metastable curves and phase transition curves for $\chi_{0}=0.01$, respectively. (c) and (d) depict the limit of the metastability curves and phase transition curves for $\chi_{0}=0.1$, respectively. In this case, all stable steady states are either uniaxial purely nematics $(N)$ or uniaxial polar nematics $(P N)$. There are coexistent regions for nematic and polar nematics labeled $N / P N$. The phase transition curve depicted in (b) and (d) are the curve along which the energies of the coexisting phases are equal.

selected values of $\alpha$. Figure 2.3 depicts the solution at $\chi_{0}=0.1$ and $\alpha=1.3$. A stable uniaxial nematic phase with a small uniaxial order parameter $s$ exists up to a critical concentration $N_{c}^{(1)}$. It then goes through a hysteresis bifurcation to yield a stable prolate uniaxial phase with a higher nematic order. At a higher concentration, $N_{c}^{(3)}$, the purely nematic phase gives way to a pair of polar nematic phases through a pitchfork bifurcation in $s_{1}$. The stable polar nematic phase remains prolate and uniaxial. This scenario persists to a critical value of $\alpha$. For instance it remains at $\alpha=1.5$. 


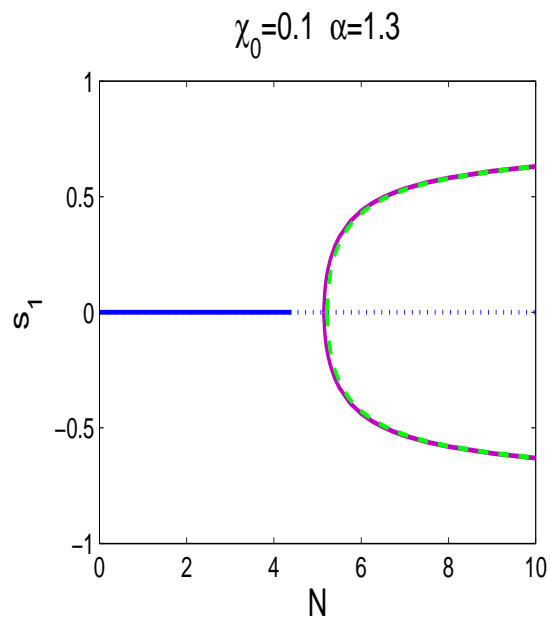

(a)

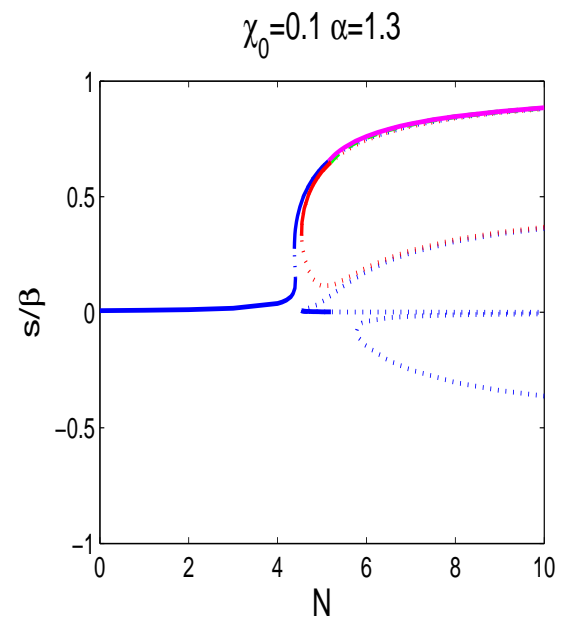

(b)

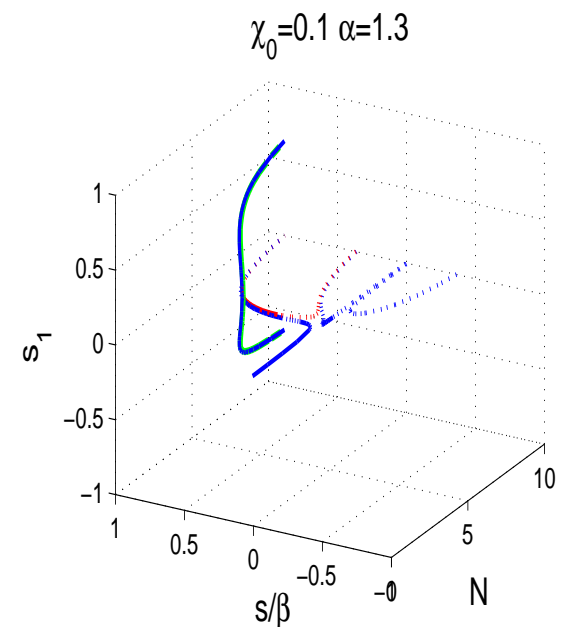

(c)

FIG. 2.3. The phase bifurcation diagram for all the order parameters in an imposed external field as functions of dimensionless concentration $N$. The parameter values are $\alpha=1.3, \chi_{0}=0.1$. The stable solutions when the polarity vector is parallel to the external field are plotted in solid curves, while the ones when the polarity vector is perpendicular to the external field are plotted in dashed curves. The stable solutions are uniaxial prolate nematics $(s>0, \beta=0)$ that are weakly aligned at low concentration and highly aligned at concentrations higher than $N_{c}^{(1)}=4.305$. The metastable biaxial states form a family of solutions parameterized by the rotational group SO(2) at high concentration whose major director is parallel to the external field direction. All others steady states plotted in other patterned curves are unstable. (a) depicts the steady states in polar order parameter $s_{1}$. (b) depicts the steady states in the uniaxial nematic order parameter $s$ for the parallel state (solid) and the biaxial order parameter $\beta$ in the perpendicular state (dashed). (c) plots the steady states in space $\left(N, s \beta, s_{1}\right)$. 


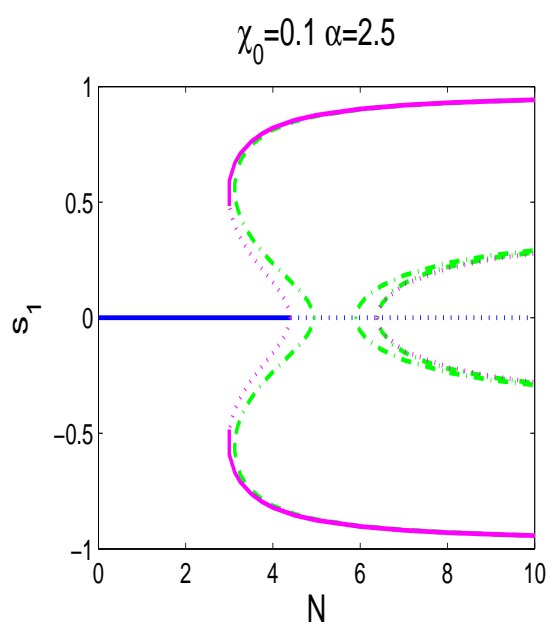

(a)

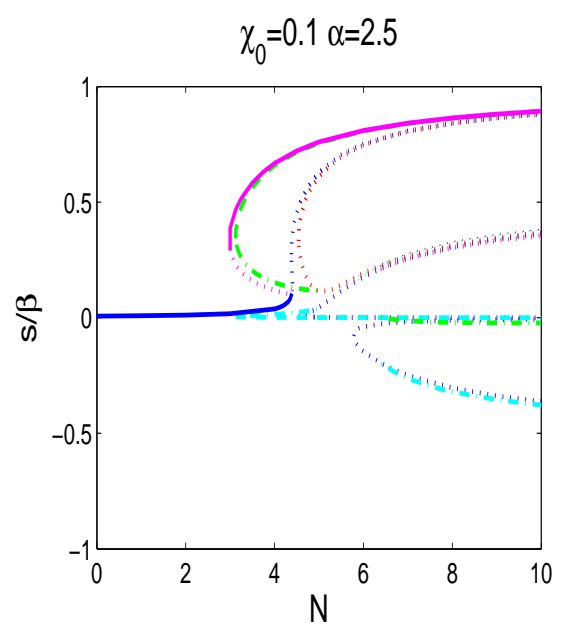

(b)

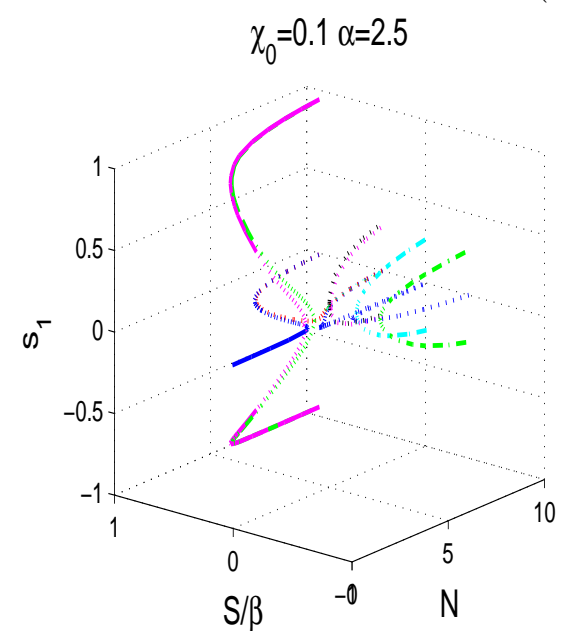

(c)

FIG. 2.4. The phase bifurcation diagram for all the order parameters in an imposed external field as functions of $N$. The parameter values are $\alpha=2.5, \chi_{0}=0.1$. The stable solutions when the polarity vector is parallel to the external field are plotted in solid curves while the other stable solutions are plotted in dashed curves. The curves of the other patterns are for unstable solutions. The stable solutions are uniaxial prolate ones $(s>0, \beta=0)$ which are weakly aligned at low concentration. $A$ stable, uniaxial, polar nematic steady state emerges beyond a critical concentration $N_{c}$ as the result of a saddle node bifurcation in the phase space $\left(N, s_{1}\right)$, with the polarity vector parallel to the external field direction. At a slightly higher critical concentration, a metastable biaxial steady state emerges also as a result of saddle node bifurcation, whose major director is parallel to the external field direction. Other steady states, uniaxial and biaxial alike, are unstable. (a) depicts the steady states in polar order parameter $s_{1}$. (b) plots the steady states in the uniaxial order parameter $s$ in parallel state (solid) and the biaxial order parameter $\beta$ in the perpendicular state (dashed). (c) plots the steady states in the phase space $\left(N, s / \beta, s_{1}\right)$. 


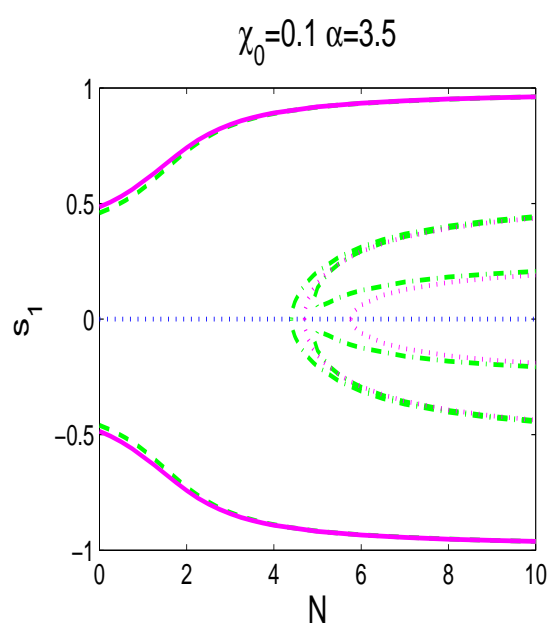

(a)

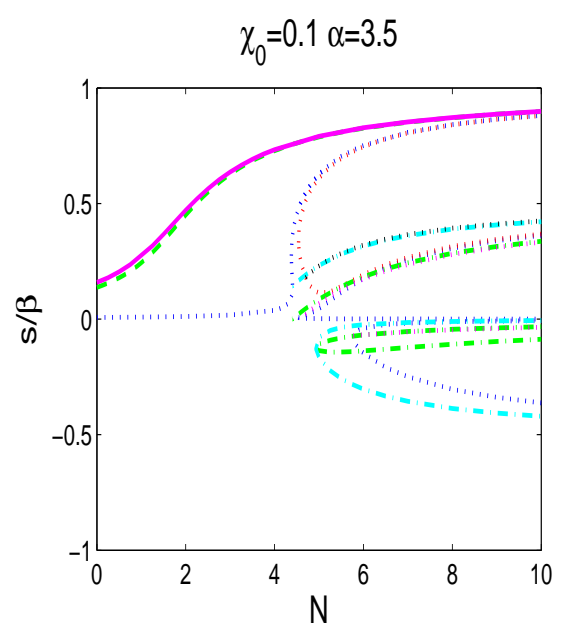

(b)

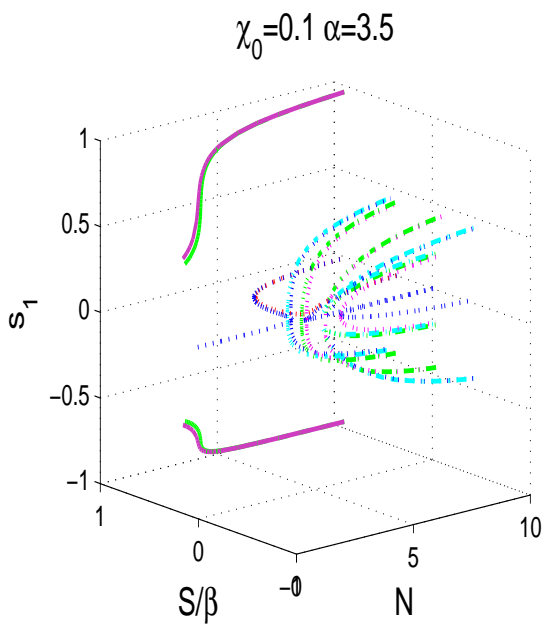

(c)

FIG. 2.5. The phase bifurcation diagram for all the order parameters in an imposed external field. The stable solutions are plotted in solid (polarity parallel to the external field) and dashed (polarity perpendicular to the external field) curves. The parameter values are $\alpha=3.5, \chi_{0}=0.1$. The globally stable solutions (solid curves) are uniaxial prolate ones $(s>0, \beta=0)$ which are highly aligned. The metastable steady states (dashed curves) are biaxial with the major director parallel to the external field direction. The others are all unstable and are plotted in curves other than solid and dashed curves. (a) depicts the steady states in polar order parameter $s_{1}$. (b) plots the steady states in the uniaxial order parameter $s$ in parallel state (solid) and the biaxial order parameter $\beta$ in the perpendicular state (dashed). (c) plots the steady states in the phase space $\left(N, s / \beta, s_{1}\right)$.

At higher strength of $\alpha$, say $\alpha=2.5$, a different scenario emerges. The stable uniaxial nematic phase gives away to a pair of unstable polar nematic phases at $N_{c}^{(1)}$, and then regains stability via saddle node bifurcations to a pair of polar nematics at lower concentrations shown in Figure 2.4. The stable polar nematic branch and the 


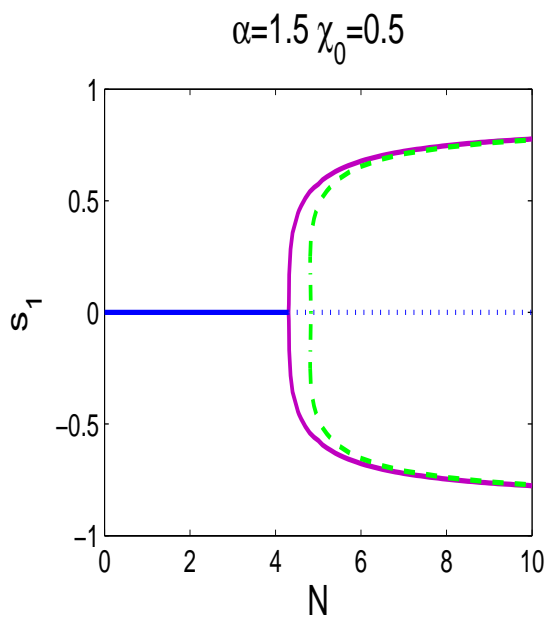

(a)

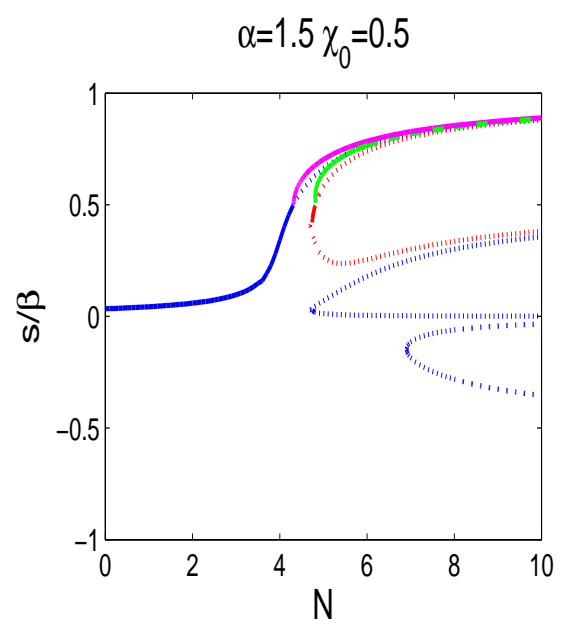

(b)

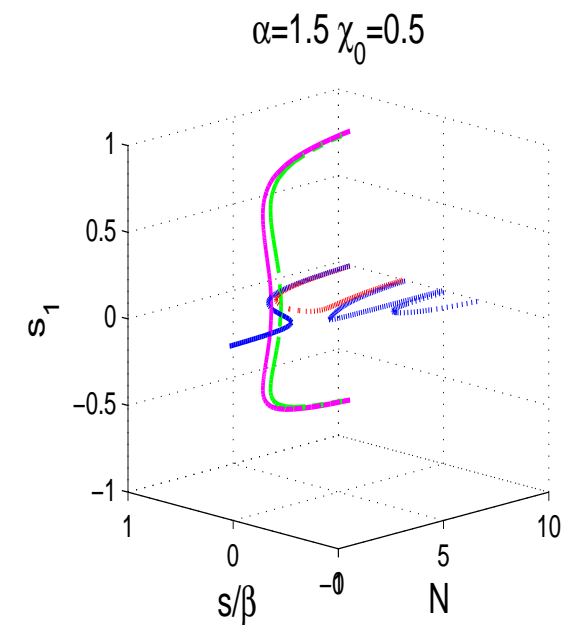

(c)

FIG. 2.6. The phase bifurcation diagram for the order parameters in an imposed external field. The parameter values are $\alpha=1.5, \chi_{0}=0.5$. The stable steady states with the polarity parallel to the external field are plotted in solid curves, while the metastable steady states with the polarity vector perpendicular to the external field are plotted in dashed ones. The globally stable steady states are uniaxial, prolate. The metastable ones are biaxial with the major director parallel to the external field direction. The biaxial nematic branch is first born purely nematic at an intermediate range of the nematic concentration $N$. Soon after it is born, a pitchfork bifurcation in the polar order parameter leads to a polar nematic phase. The other steady states, uniaxial and biaxial alike, are unstable. (a) depicts the steady states in the polar order parameter $s_{1}$. (b) plots the steady states in the uniaxial order parameter s in parallel state (solid) and the biaxial order parameter $\beta$ in the perpendicular state (dashed). (c) plots the steady states in the phase space $\left(N, s / \beta, s_{1}\right)$. The increase in $\chi_{0}$ results in the delay of the formation of metastable biaxial polar nematics to the higher concentration. 


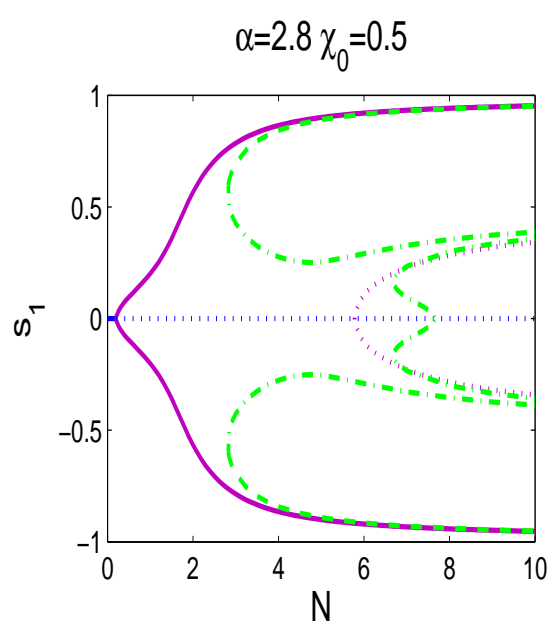

(a)

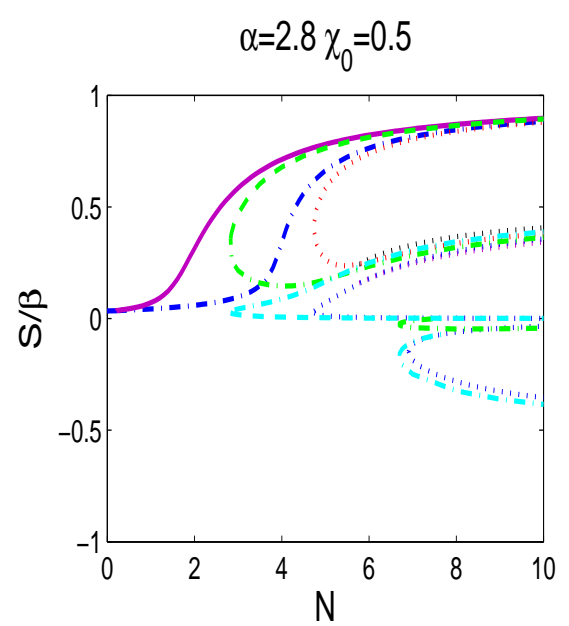

(b)

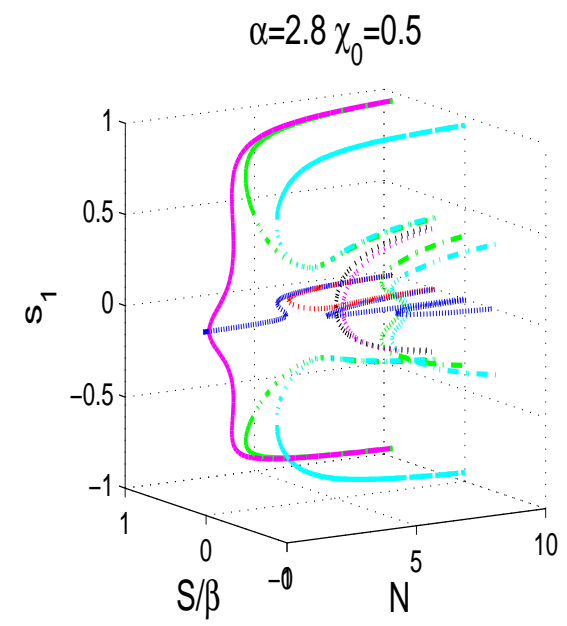

(c)

FIG. 2.7. The phase bifurcation diagram for all the order parameters in an imposed field. The parameter values are $\alpha=2.8, \chi_{0}=0.5$. The stable solutions are plotted in solid (parallel polarity) and dashed (perpendicular polarity) curves. The stable steady states in the case where the polarity is parallel to the external field are uniaxial prolate $(s>0, \beta=0)$ and highly aligned. At high concentration, a metastable biaxial steady state emerges with the polarity vector perpendicular to the external field. All other curves represent unstable steady states. (a) depicts the steady states in polar order parameter $s_{1}$. (b) plots the steady states in the uniaxial order parameter $s$ in parallel state (solid) and the biaxial order parameter $\beta$ in the perpendicular state (dashed). (c) plots the steady states in the phase space $\left(N, s / \beta, s_{1}\right)$.

unstable one connected to it remain prolate and uniaxial. This persists through $\alpha=3$. For $\alpha>3$, the prolate, uniaxial polar nematic exists beginning at $N=0$ and becomes the only stable state. See Figure 2.5. We remark that the polar nematic steady states exist in pairs with the polar order $\pm s_{1}$. The solution behavior changes slightly when 


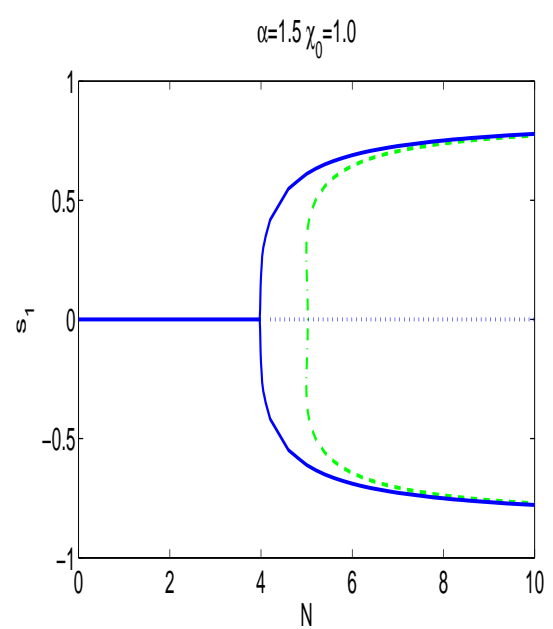

(a)

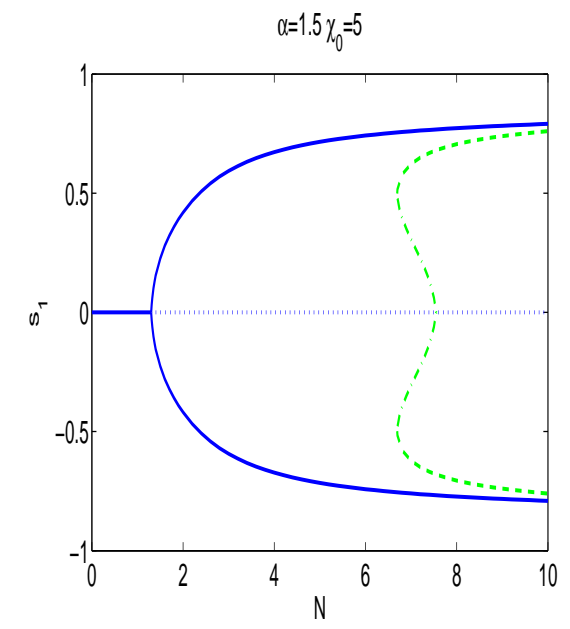

(c)

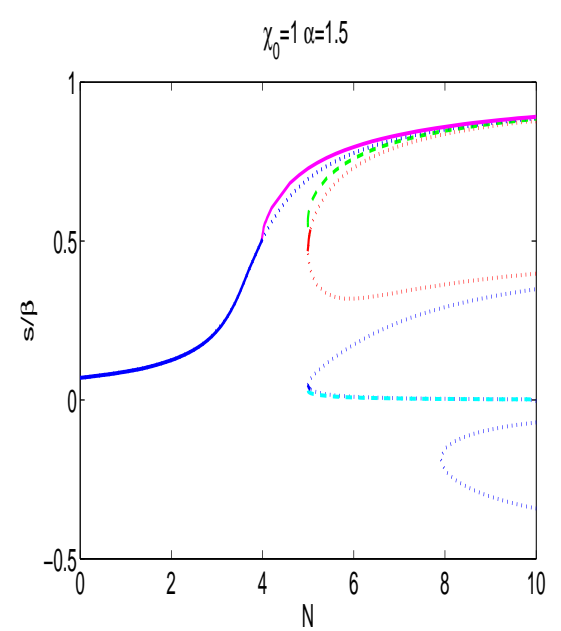

(b)

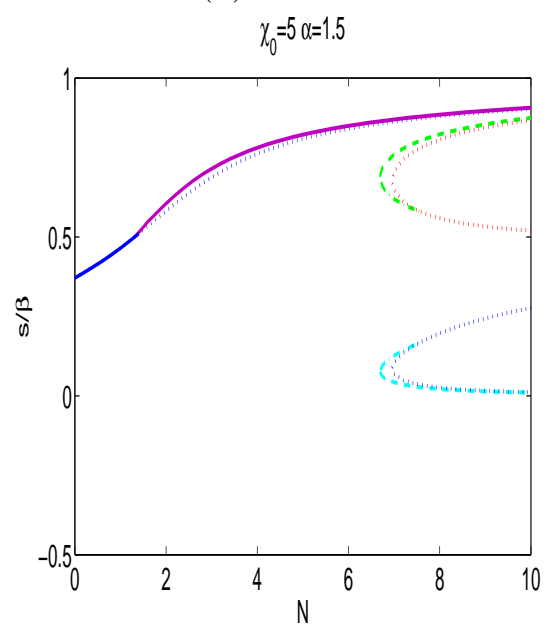

FIG. 2.8. The polar order parameter $s_{1}$ as well as the nematic order parameters $s / \beta$ at $\alpha=1.5$ and $\chi_{0}=1,5$. (a) and (b) depict the order parameters at $\chi_{0}=1$ and (c) and (d) depict the order parameters at $\chi_{0}=5$. The effect of $\chi_{0}$ in the steady state with parallel polarity is to promote the formation of the polar order at low concentration, whereas the effect of $\chi_{0}$ in the state with perpendicular polarity is to delay the formation of the metastable nematic order to higher concentration.

$\chi_{0}$ increases to $\chi_{0}=0.5$. The hysteresis bifurcation along the purely nematic branch disappears. However, the pitchfork, a transcritical bifurcation, persists, yielding the pair of polar nematics. Figure 2.6 and 2.7 depict the solution behavior at $\alpha=1.5,2.8$. When $\alpha>3$, the behavior is analogous to what is depicted in Figure 2.5. When $\chi_{0}$ is increased further, the general trend is that the nematic order increases and the critical concentration and strength of dipole potential decreases. Figure 2.8 depicts two steady states at $\alpha=1.5$ and $\chi_{0}=1,5$, respectively. At the larger value of $\chi_{0}$, the 
critical concentration where the polar order emerges decreases below $N=2$. The other features alluded to for smaller $\chi_{0}$ remain.

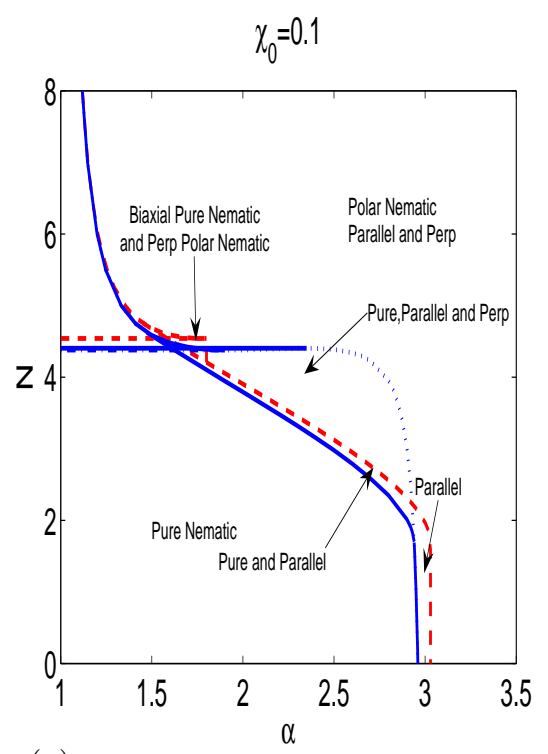

(a)

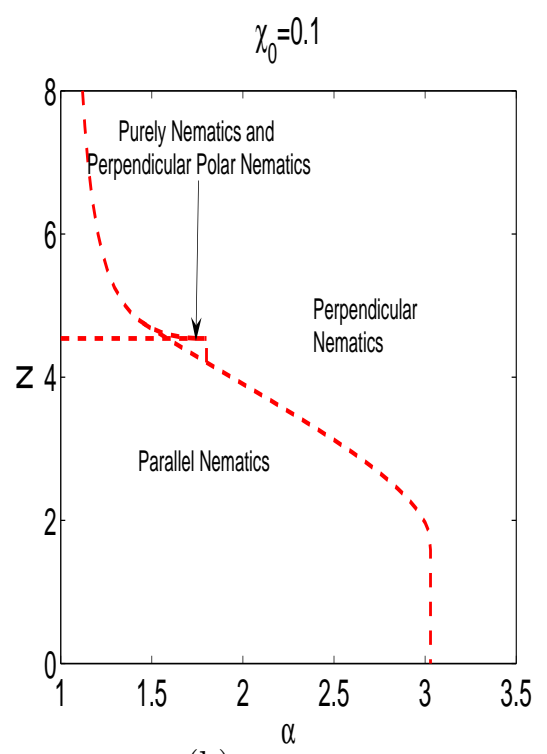

(b)

FIG. 2.9. The phase diagram for extended nematics in an imposed magnetic field at $\chi_{0}=0.1$. Both steady states of polarity parallel and perpendicular to the external field are included. (a) depicts the limits of the metastable curves, while (b) depicts the limit curves for the steady states with the perpendicular polarity. Parallel nematics mean the uniaxial steady states with the polarity parallel to the external field, while the perpendicular nematics imply the biaxial steady states with the polarity perpendicular to the external field. The curves plotted in (b) are part of those in (a). This gives all the possible phases in parameter space $(\alpha, N)$.

2.3. Concentrated limit: steady states with the polarity vector perpendicular to the external field. From the limiting case $N=0$, we learned that there exist biaxial steady states when the polarity vector is perpendicular to the imposed external field direction; however, these are unstable. In [38], we showed that stable biaxial nematic steady states can exist when excluded volume and external field potential are coupled. In the dipole-dipole interaction, the excluded volume interaction and the external field are all coupled, we show numerically that the biaxial state can be stabilized in the non-dilute limit by the excluded volume potential, but these steady states are only metastable.

In the weak external field, Figure 2.3 shows the phase transition of a stable purely nematic biaxial steady state to a pair of biaxial, polar nematic phases through a pitchfork bifurcation in the polar order parameter given by the dashed curves. The stable biaxial phase is borne out of a saddle node bifurcation with one branch metastable at intermediate concentration $N$. The existence of the metastable purely nematic biaxial state is reminiscent of the similar steady state reported in [38] without the dipole-dipole interaction. We note, however, that the stable biaxial steady state is nearly uniaxial with the major director in the direction of the external field! This scenario persists to a critical value of $\alpha$. In Figure $2.4(\alpha=2.5)$, the biaxial steady state is born stable and polar through a saddle node bifurcation at lower concentrations. For $\alpha>3$, the stable biaxial polar nematic phase exists for all $N>0$. The dashed curves 
in these figures represent the stable steady states corresponding to the perpendicular external field. So the effect of a perpendicular external field is to induce metastable, biaxial steady states with their major director aligned in the external field direction. As the strength of the external field increases, i.e., as $\chi_{0}$ increases, the formation of the polar nematic phase is pushed to a higher concentration at fixed $\alpha$ and the metastable biaxial nematic phase tends to be born polar in this range of $\chi_{0}$. See Figure 2.8 for the case of $\alpha=1.5$ and $\chi_{0}=1,5$. So, in high external fields, the metastable biaxial, steady state survives only in high-concentration regions as polar nematic. The phase diagram in parameter space $(\alpha, N)$ for the perpendicular external field at $\chi_{0}=0.1$ is given in Figure 2.9, where the dotted curves define the limits separating the biaxial polar nematics from the other nematics. We note the existence of a coexistence region where pure nematics and perpendicular polar nematics coexist. The region labeled parallel nematics is the one where nematic solutions exist only when the polarity vector is parallel to the external field.

We next investigate the extended nematics with permanent dipoles (or magnetic moments).

\section{Extended Nematics with permanent dipoles $(\mu \neq 0)$}

We first establish a lemma to reveal the relation among the nematic order tensor, the polarity vector and the external field in this case.

Lemma 3.1. In steady states, there exists a constant $c$ such that $\mu\langle\mathbf{m}\rangle+\chi_{0} \mathbf{M} \cdot \mathbf{E}=$ $c \mathbf{E}$.

Proof. From the introduction,

$$
\left\langle\mathcal{R} V_{e}\right\rangle=0
$$

so we have

$$
\mu\langle\mathbf{m}\rangle \times \mathbf{E}+\chi_{0} \mathbf{M} \cdot \mathbf{E} \times \mathbf{E}=0 .
$$

This implies

$$
\mu\langle\mathbf{m}\rangle+\chi_{0} \mathbf{M} \cdot \mathbf{E}|| \mathbf{E} .
$$

Namely, there exists a constant $c$ such that

$$
\mu\langle\mathbf{m}\rangle+\chi_{0} \mathbf{M} \cdot \mathbf{E}=c \mathbf{E}
$$

With this lemma, we can establish the following theorem.

TheOREM 3.1. When $\mu \neq 0$, the polarity vector is nonzero in steady states. Thereby, the steady states are all polar.

Proof. We show this by a counterproof. Suppose $\langle\mathbf{m}\rangle=0$; the lemma implies $\mathbf{E}$ is an eigenvector of the second moment. We choose the coordinates such that the second moment is diagonal and $\mathbf{E}=E(0,0,1)$. We denote

$$
\begin{aligned}
& F(\lambda)=\int_{\|\mathbf{m}\|=1} m_{3} e^{h(\lambda)} d \mathbf{m}, \\
& h(\lambda)=\lambda \mu E m_{3}+\frac{3 N}{2} \sum_{i=1}^{3} M_{i i} m_{i}^{2}+\frac{\chi_{0}}{2} E^{2} m_{3}^{2} .
\end{aligned}
$$


Then $\left\langle m_{3}\right\rangle=F(1)=0$ and $F^{\prime}(\lambda)>0$. It can be easily verified that $F(0)=0$. Thus, there exists a $0<\lambda^{*}<1$ such that

$$
F^{\prime}\left(\lambda^{*}\right)=\mu E \int_{\|\mathbf{m}\|=1} m_{3}^{2} e^{h} d \mathbf{m}=0
$$

This contradicts $F^{\prime}(\lambda)>0$. The polarity vector must be nonzero.

Analogous to the results established in the previous section, we can show the next result.

THEOREM 3.2. When $\alpha \leq 1$, the nonzero polarity vector and the external field must be parallel to each other.

Proof. We assume $\mathbf{E}=E(0,0,1)$ and $\langle\mathbf{m}\rangle=\left(r_{1}, 0, r_{3}\right)$ with $r_{1}>0$. Then,

$$
f=\frac{1}{Z} e^{\alpha r_{1} m_{1}+V_{2}\left(m_{1}, m_{2}, m_{3}\right)}
$$

where $V\left(m_{1}, m_{2}, m_{3}\right)$ is even in $m_{1}$.

$$
\begin{aligned}
r_{1} & =\left\langle m_{1}\right\rangle=\frac{\int_{\|\mathbf{m}\|=1} m_{1} f d \mathbf{m}}{\int_{\|\mathbf{m}\|=1} f d \mathbf{m}}=\frac{\int_{\|\mathbf{m}\|=1, m_{1}>0} m_{1} \sinh \left(\alpha r_{1} m_{1}\right) \exp \left[V_{2}\left(m_{1}, m_{2}, m_{3}\right)\right] d \mathbf{m}}{\int_{\|\mathbf{m}\|=1, m_{1}>0} \cosh \left(\alpha r_{1} m_{1}\right) \exp \left[V_{2}\left(m_{1}, m_{2}, m_{3}\right)\right] d \mathbf{m}} \\
& \leq \alpha r_{1} \frac{\int_{\|\mathbf{m}\|=1, m_{1}>0} m_{1} \cosh \left(\alpha r_{1} m_{1}\right) \exp \left[V_{2}\left(m_{1}, m_{2}, m_{3}\right)\right] d \mathbf{m}}{\int_{\|\mathbf{m}\|=1, m_{1}>0} \cosh \left(\alpha r_{1} m_{1}\right) \exp \left[V_{2}\left(m_{1}, m_{2}, m_{3}\right)\right] d \mathbf{m}}<\alpha r_{1} .
\end{aligned}
$$

When $\alpha \leq 1, r_{1}=0$. Namely, the polarity vector is parallel to the external field.

A simple argument given below rules out the possibility of the existence of a steady state whose polarity vector is perpendicular to the external field. Let's assume $r_{3}=$ $\left\langle m_{3}\right\rangle=0$ in the steady state. Then, a simple calculation shows

$$
\begin{aligned}
r_{3} & =\left\langle m_{3}\right\rangle=\frac{1}{Z} \int_{\|\mathbf{m}\|=1} m_{3} \exp \left[\mu m_{3}+\alpha r_{1} m_{1}+\frac{3 N}{2} \sum_{i=1}^{3} M_{i i} m_{i}^{2}+\frac{\chi_{0}}{2} E^{2} m_{3}^{2}\right] d \mathbf{m} \\
& =\frac{2}{Z} \int_{\|\mathbf{m}\|=1, m_{3}>0} m_{3} \sinh \left(\mu m_{3}\right) \exp \left[\alpha r_{1} m_{1}+\frac{3 N}{2} \sum_{i=1}^{3} M_{i i} m_{i}^{2}+\frac{\chi_{0}}{2} E^{2} m_{3}^{2}\right] d \mathbf{m} \neq 0 .
\end{aligned}
$$

Thus, the polarity vector will never be perpendicular to the external field in steady states.

In addition, we can show that the polarity vector and the external field are parallel in certain parameter regimes given below.

TheOREM 3.3. When $|\mu|>\chi_{0} E$ and $3 N \mu<\left|\alpha \chi_{0} E\right|$, the polarity vector and the electric field are parallel to each other and are both eigenvectors of the nematic order tensor.

Proof. We select the $z$-axis to be the direction of $\mathbf{E}$ and the $y$-axis to be perpendicular to the plane spanned by $\langle\mathbf{m}\rangle$ and $\mathbf{E}$. If $\langle\mathbf{m}\rangle$ and $\mathbf{E}$ are parallel to each other, they must be one of the eigenvectors of the second moment by the lemma. We 
therefore focus on the case where they are not parallel to each other. In this Cartesian coordinate system, we have

$$
\begin{aligned}
\mathbf{m} & =\left(m_{1}, m_{2}, m_{3}\right), \mathbf{E}=E(0,0,1),\langle\mathbf{m}\rangle=\left(r_{1}, 0, r_{3}\right), \\
U(\mathbf{m}) & =-\alpha r_{1} m_{1}-\left(\mu E+\alpha r_{3}\right) m_{3}-\frac{3 N}{2} \mathbf{M}: \mathbf{m m}-\frac{\chi_{0}}{2} E^{2} m_{3}^{2}, \\
f(\mathbf{m}) & =\frac{1}{Z} \exp \left[\alpha r_{1} m_{1}+\left(\mu E+\alpha r_{3}\right) m_{3}+\frac{3 N}{2} \mathbf{M}: \mathbf{m m}+\frac{\chi_{0}}{2} E^{2} m_{3}^{2}\right], \\
Z & =\int_{S} \exp \left[\alpha r_{1} m_{1}+\left(\mu E+\alpha r_{3}\right) m_{3}+\frac{3 N}{2} \mathbf{M}: \mathbf{m m}+\frac{\chi_{0}}{2} E^{2} m_{3}^{2}\right] d \mathbf{m} .
\end{aligned}
$$

Note that our choice of the Cartesian coordinate system makes $E$ nonnegative and $M_{23}=0$ from the lemma. We also select the positive direction of the polarity vector so that $\mu$ is always nonnegative. The nonlinear integral equations for $r_{1}$ and $r_{3}$ are

$$
\begin{aligned}
& r_{1}=\left\langle m_{1}\right\rangle=\int_{S} m_{1} f(\mathbf{m}) d \mathbf{m}, \\
& r_{3}=\left\langle m_{3}\right\rangle=\int_{S} m_{3} f(\mathbf{m}) d \mathbf{m} .
\end{aligned}
$$

We use the spherical coordinate system where the pole is the $y$-axis and we denote it by $(\psi, \zeta)$. We have

$$
\begin{aligned}
\left(m_{1}, m_{2}, m_{3}\right)= & (\sin \psi \sin \zeta, \cos \psi, \sin \psi \cos \zeta), \\
U(\psi, \zeta)= & -\alpha r_{1} \sin \psi \sin \zeta-\left(\mu E+\alpha r_{3}\right) \sin \psi \cos \zeta-\frac{\chi_{0}}{2} E^{2} \sin ^{2} \psi \cos ^{2} \zeta \\
& -\frac{3 N}{2}\left(M_{11} \sin ^{2} \psi \sin ^{2} \zeta+M_{22} \cos ^{2} \psi+M_{33} \sin ^{2} \psi \cos ^{2} \zeta\right. \\
& \left.+2 M_{12} \sin \psi \cos \psi \sin \zeta+M_{13} \sin ^{2} \psi \sin 2 \zeta\right) \\
= & U_{\mathrm{Mut}}(\psi, \zeta)+U_{\mathrm{Ext}}(\psi, \zeta),
\end{aligned}
$$

where the mutual interaction and the external interaction parts of the potential are

$$
\begin{aligned}
U_{\mathrm{Mut}}(\psi, \zeta)= & -\alpha r_{1} \sin \psi \sin \zeta-\alpha r_{3} \sin \psi \cos \zeta \\
& -\frac{3 N}{2}\left(M_{11} \sin ^{2} \psi \sin ^{2} \zeta+M_{22} \cos ^{2} \psi+M_{33} \sin ^{2} \psi \cos ^{2} \zeta\right. \\
& \left.+2 M_{12} \sin \psi \cos \psi \sin \zeta+M_{13} \sin ^{2} \psi \sin 2 \zeta\right), \\
U_{\mathrm{Ext}}(\psi, \zeta)= & -\mu E \sin \psi \cos \zeta-\frac{\chi_{0}}{2} E^{2} \sin ^{2} \psi \cos ^{2} \zeta .
\end{aligned}
$$

$r_{1}$ and $r_{3}$ are given by

$$
\begin{aligned}
& r_{1}=<\sin \psi \sin \zeta>=\int_{0}^{\pi} \int_{0}^{2 \pi} \sin \psi \sin \zeta f(\psi, \zeta) d \psi \sin \psi d \zeta \\
& r_{3}=<\sin \psi \cos \zeta>=\int_{0}^{\pi} \int_{0}^{2 \pi} \sin \psi \cos \zeta f(\psi, \zeta) d \psi \sin \psi d \zeta .
\end{aligned}
$$

We need to prove that all solutions of (3.11) satisfy $r_{1}=0$. We prove it by contradiction. Suppose there is a solution of (3.11) satisfying $r_{1} \neq 0$. It is straightforward to show that if $\left(r_{1}, r_{3}\right)$ is a solution of $(3.11)$, then $\left(-r_{1}, r_{3}\right)$ is also a solution of (3.11). 
Without loss of generality, we assume $r_{1}>0$. We are going to show that $\mu \geq \chi_{0} E$ and $r_{1}>0$ lead to $\left\langle\frac{\partial}{\partial \zeta} U_{\text {Ext }}(\psi, \zeta)\right\rangle>0$, which contradicts $\left\langle\frac{\partial U}{\partial \zeta}\right\rangle=0$ proved in [42]. In fact $\left\langle\frac{\partial U}{\partial \zeta}\right\rangle=0$ implies $\left\langle\frac{\partial U_{e x t}}{\partial \zeta}\right\rangle=0$.

Noticing that $U_{\mathrm{Ext}}(\psi, \zeta)$ is an even function of $\zeta$ and consequently $\frac{\partial}{\partial \zeta} U_{\mathrm{Ext}}(\psi, \zeta)$ is an odd function of $\zeta$, we have

$$
\left\langle\frac{\partial}{\partial \zeta} U_{\operatorname{Ext}}(\psi, \zeta)\right\rangle=\int_{0}^{\pi} \int_{0}^{\pi} \frac{\partial}{\partial \zeta} U_{\operatorname{Ext}}(\psi, \zeta)\{\exp [-U(\psi, \zeta)]-\exp [-U(\psi,-\zeta)]\} d \zeta \sin \psi d \psi
$$

In the above, the first factor of the integrand satisfies

$$
\begin{aligned}
\frac{\partial}{\partial \zeta} U_{\mathrm{Ext}}(\psi, \zeta) & =\mu E \sin \psi \sin \zeta+\chi_{0} E^{2} \sin ^{2} \psi \cos \zeta \sin \zeta \\
& =\left(\mu+\chi_{0} E \sin \psi \cos \zeta\right) E \sin \psi \sin \zeta, \text { for } \zeta \in(0, \pi), \psi \in(0, \pi)
\end{aligned}
$$

The second factor of the integrand satisfies, for $\zeta \in(0, \pi), \psi \in(0, \pi)$,

$$
\begin{gathered}
\exp [-U(\psi, \zeta)]-\exp [-U(\psi,-\zeta)] \\
=2 \exp \left[\frac{\chi_{0}}{2} E^{2} \sin ^{2} \psi \cos ^{2} \zeta+\left(\mu E+\alpha r_{3}\right) \sin \psi \cos \zeta+\frac{3 N}{2} \sum_{i=1}^{3} M_{i i} m_{i}^{2}\right] \\
\sinh \left(\alpha r_{1} \sin \psi \sin \zeta+3 N M_{12} \sin \psi \cos \psi \sin \zeta+\frac{3 N}{2} M_{13} \sin ^{2} \psi \sin 2 \zeta\right)
\end{gathered}
$$

Introducing the change of variable $\psi \rightarrow \pi-\psi$ while $\psi \in[\pi / 2, \pi]$, the integral reduces to

$$
\begin{array}{r}
\left\langle\frac{\partial}{\partial \zeta} U_{\operatorname{Ext}}(\psi, \zeta)\right\rangle=4 \int_{0}^{\pi / 2} \int_{0}^{\pi} \frac{\partial}{\partial \zeta} U_{\operatorname{Ext}}(\psi, \zeta) \exp \left[\frac{\chi_{0}}{2} E^{2} \sin ^{2} \psi \cos ^{2} \zeta+\right. \\
\left.\left(\mu E+\alpha r_{3}\right) \sin \psi \cos \zeta+\frac{3 N}{2} \sum_{i=1}^{3} M_{i i} m_{i}^{2}\right]
\end{array}
$$

$\sinh \left(\alpha r_{1} \sin \psi \sin \zeta+\frac{3 N}{2} M_{13} \sin ^{2} \psi \sin 2 \zeta\right) \cosh \left(3 N M_{12} \sin \psi \cos \psi \sin \zeta\right) d \psi d \zeta$.

From the lemma, we have

$$
\begin{gathered}
\mu r_{1}=-\chi_{0} E M_{13} . \\
\alpha r_{1} \sin \psi \sin \zeta+\frac{3 N}{2} M_{13} \sin ^{2} \psi \sin 2 \zeta=M_{13} \sin \psi \sin \zeta\left(-\frac{\alpha \chi_{0}}{\mu} E+3 N \sin \psi \cos \zeta\right) .
\end{gathered}
$$

When $\mu>\left|\chi_{0} E\right|$ and $3 N \mu<\left|\alpha \chi_{0} E\right|$, the above integral cannot be zero unless $r_{1}=$ $M_{13}=0$. Therefore, the direction of $\langle\mathbf{m}\rangle$ must be parallel to that of $\mathbf{E}$. When these two vectors are parallel to each other, the lemma implies that they must be one of the eigenvectors of the nematic order tensor. 
REMARK 3.4. The inequalities in the above theorem also imply that $\alpha>3 N$.

When the condition in the theorem is violated, nonparallel solutions may exist. Without loss of generality, we set $\mathbf{E}=E(0,0,1),\langle\mathbf{m}\rangle=\left(r_{1}, 0, r_{3}\right)$, and parameterize

$$
\mathbf{m}=(\sin \theta \cos \phi, \sin \theta \sin \phi, \cos \theta) .
$$

Then,

$$
\begin{aligned}
& f=\frac{1}{Z} e^{h}, \\
& h=\mu E \cos \theta+\alpha\left(r_{1} \sin \theta \cos \phi+r_{3} \cos \theta\right)+\frac{3 N}{2}\left[M_{11} \sin ^{2} \theta \cos ^{2} \phi+M_{12} \sin ^{2} \theta \sin 2 \phi+\right. \\
& \left.\quad M_{13} \sin 2 \theta \cos \phi+M_{22} \sin ^{2} \theta \sin ^{2} \phi+M_{33} \cos ^{2} \theta\right]+\frac{\chi_{0} E^{2}}{2} \cos ^{2} \theta, \\
& r_{1}=\langle\sin \theta \cos \phi\rangle, r_{3}=\langle\cos \theta\rangle, M_{11}=\left\langle\sin ^{2} \theta \cos ^{2} \phi\right\rangle, M_{12}=\frac{1}{2}\left\langle\sin ^{2} \theta \sin 2 \phi\right\rangle, \\
& M_{13}=\frac{1}{2}\langle\sin 2 \theta \cos \phi\rangle, M_{22}=\left\langle\sin ^{2} \theta \sin ^{2} \phi\right\rangle, M_{33}=\left\langle\cos ^{2} \theta\right\rangle .
\end{aligned}
$$

The pdf steady state solution can further be simplified in the case of $r_{1} \neq 0$. The following lemma shows that $M_{12}=0$ if $r_{1} \neq 0$ indicating that nonparallel steady states are in plane with the polarity vector and the external field.

Lemma 3.2. When $\left\langle m_{2}\right\rangle=M_{23}=0,\left\langle m_{1}\right\rangle M_{12}=0$.

Proof. We note that the Cartesian coordinate and the spherical coordinate are related by the following relations:

$$
\begin{array}{lll}
x_{1}=\rho \sin \theta \cos \phi, & x_{2}=\rho \sin \theta \sin \phi, & x_{3}=\rho \cos \theta, \\
\frac{\partial \rho}{\partial x_{1}}=\sin \theta \cos \phi, & \frac{\partial \rho}{\partial x_{2}}=\sin \theta \sin \phi, & \frac{\partial \rho}{\partial x_{3}}=\cos \theta, \\
\frac{\partial \theta}{\partial x_{1}}=\frac{1}{\rho} \cos \theta \cos \phi, & \frac{\partial \theta}{\partial x_{2}}=\frac{1}{\rho} \cos \theta \sin \phi, & \frac{\partial \theta}{\partial x_{3}}=-\frac{1}{\rho} \sin \theta, \\
\frac{\partial \phi}{\partial x_{1}}=-\frac{\sin \phi}{\rho \sin \theta}, & \frac{\partial \phi}{\partial x_{2}}=\frac{\cos \phi}{\rho \sin \theta}, & \frac{\partial \phi}{\partial x_{3}}=0 .
\end{array}
$$

It follows that

$$
\begin{aligned}
\int_{\|\mathbf{m}\|=1} \frac{\partial f}{\partial m_{2}} d \mathbf{m} & =\int_{0}^{2 \pi} d \phi \int_{0}^{\pi}\left(\frac{\partial f}{\partial \theta} \cdot \frac{\partial \theta}{\partial m_{2}}+\frac{\partial f}{\partial \phi} \cdot \frac{\partial \phi}{\partial m_{2}}\right) \sin \theta d \theta \\
& =\int_{0}^{2 \pi} d \phi \int_{0}^{\pi}\left(\sin \theta \cos \theta \sin \phi \frac{\partial f}{\partial \theta}+\cos \phi \frac{\partial f}{\partial \phi}\right) d \theta \\
& =2\left\langle m_{2}\right\rangle+\int_{0}^{\pi} d \theta \int_{0}^{2 \pi} \frac{\partial(f \cos \phi)}{\partial \phi} d \phi=2\left\langle m_{2}\right\rangle .
\end{aligned}
$$

If $\left\langle m_{2}\right\rangle=0,\left\langle m_{2} m_{3}\right\rangle=0$, the pdf has the following form:

$$
\begin{aligned}
f= & \frac{1}{Z} e^{h}, \\
h= & \alpha\left(r_{1} m_{1}+r_{3} m_{3}\right)+\mu m_{3}+\frac{\chi_{0}}{2} m_{3}^{2} \\
& +\frac{3 N}{2}\left[M_{11} m_{1}^{2}+M_{22} m_{2}^{2}+\left(1-M_{11}-M_{22}\right) m_{3}^{2}+2 M_{12} m_{1} m_{2}+2 M_{13} m_{1} m_{3}\right] .
\end{aligned}
$$


Then,

$$
\frac{\partial f}{\partial m_{2}}=3 N\left(M_{22} m_{2}+M_{12} m_{1}\right) f
$$

So we have

$$
3 N M_{22}\left\langle m_{2}\right\rangle+3 N M_{12}\left\langle m_{1}\right\rangle=2\left\langle m_{2}\right\rangle
$$

i.e., $r_{1} M_{12}=0$.

We next examine the possible nonparallel steady states and their stability in the dilute and concentrated limit, respectively.
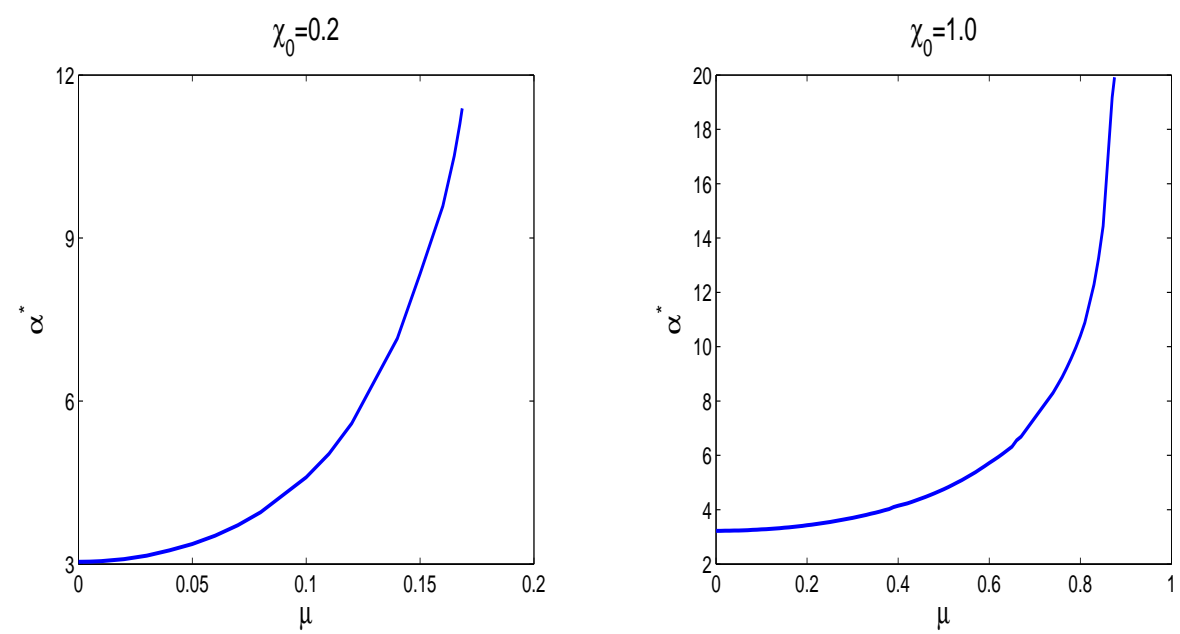

FIG. 3.1. The critical curves of $\alpha^{*}$ versus $\mu$ for $\chi_{0}=0.2,1$. On the left of the curves, the steady state polarity vector parallels the external field. Nonparallel steady states may emerge on the right of the curves.

3.1. Dilute limit. When $N=0$, the pdf solution is parameterized by $r_{1}, r_{3}$, which are determined by the solutions of

$$
r_{1}=\langle\sin \theta \cos \phi\rangle, r_{3}=\langle\cos \theta\rangle .
$$

The parallel versus nonparallel scenario corresponds to $r_{1}=0$ vs $r_{1} \neq 0$. This is studied exhaustively in [42]. It is shown that the polarity vector is parallel to the external field when $\mu \geq\left|\chi_{0} E\right|$. In addition, it is shown that there exists an $\alpha^{*}$ such that when $\alpha>\alpha^{*}$ and $\mu<\left|\chi_{0} E\right|$ a nonparallel solution exists. Of course, the condition $\alpha\left(\chi_{0} E+\mu r_{3}\right) \geq 0$ must be met if the nonparallel solution has $r_{1}>0$.

The bifurcation of $r_{1}$ away from zero is accomplished through a pitchfork bifurcation in $r_{1}$ as $\alpha \geq 1$ varies. The Jacobian of the two nonlinear equations defining $r_{1}, r_{2}$ is given by

$$
J=\left(\begin{array}{rr}
1-\alpha\left\langle\sin ^{2} \theta \cos ^{2} \phi\right\rangle & -\alpha\langle\sin \theta \cos \theta \cos \phi\rangle \\
-\alpha\langle\sin \theta \cos \theta \cos \phi\rangle & 1-\alpha\left\langle\cos ^{2} \theta\right\rangle
\end{array}\right) .
$$


When $r_{1}=0,\langle\sin \theta \cos \theta \cos \phi\rangle=0$. At the bifurcating point, $1-\alpha\left\langle\sin ^{2} \theta \cos ^{2} \phi\right\rangle=0$. This defines the critical strength $\alpha^{*}$ where the polarity vector begins to be nonparallel. Figure 3.1 depicts a set of critical curves for selected values of $\chi_{0}=0.2$ and 1 , respectively. The critical curve in $\left(\mu, \alpha^{*}\right)$ shifts to higher values in $\alpha^{*}$ when $\left|\chi_{0}\right|$ increases.
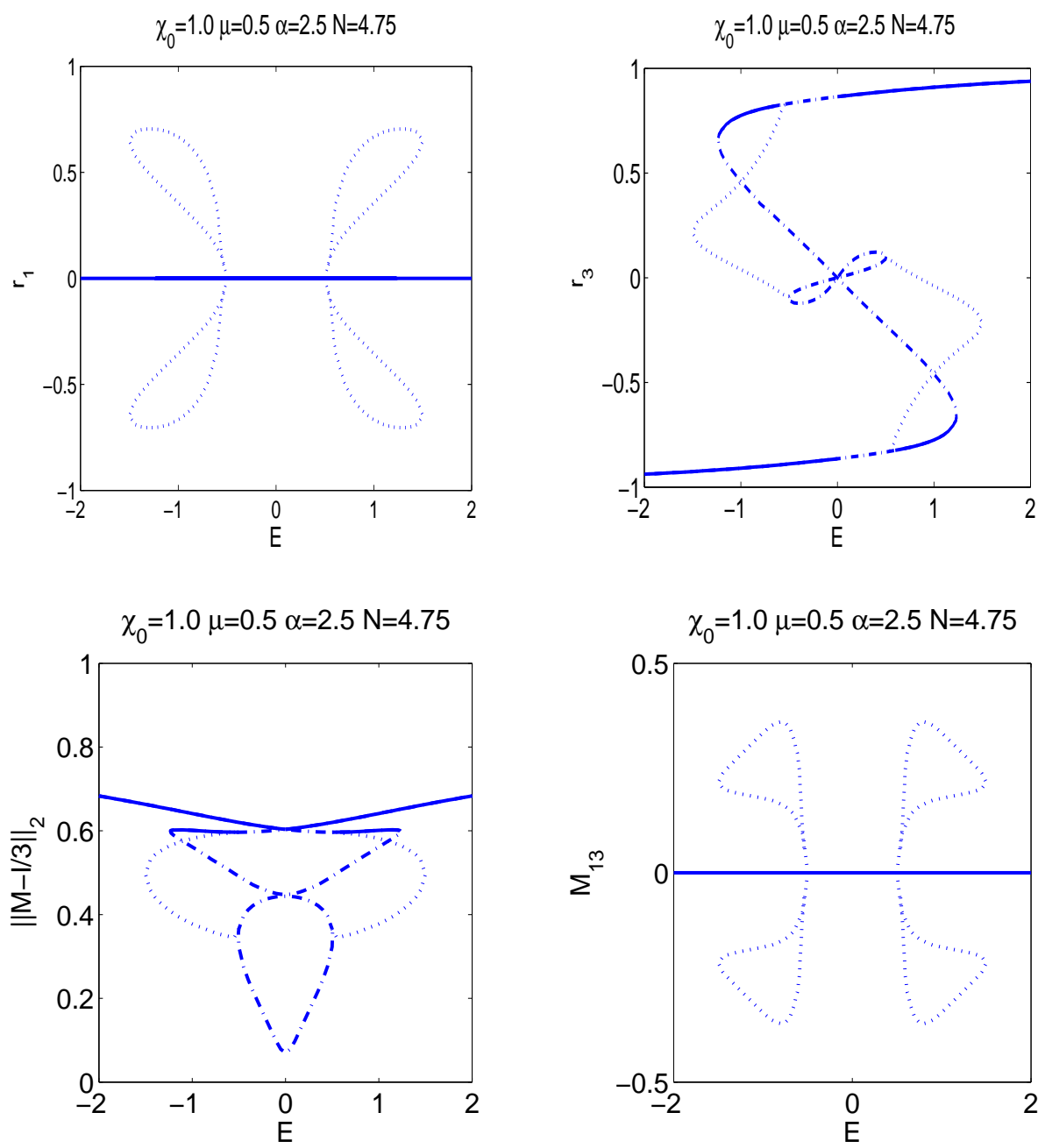

FIG. 3.2. The polarity vector as well as the nematic order tensor components in concentrated regimes. The parameter values are $\chi_{0}=1, \alpha=2.5, N=4.75, \mu=0.5$. The stable and metastable steady states are uniaxial, prolate, polar nematics with the polarity vector parallel to the external field. A metastable state can exist at intermediate range of the field strength with the polarity axis opposite to the external field direction. The nonparallel steady states in which the polarity is not parallel to the external field can exist, but are unstable. The nematic order of the globally stable steady state is higher than that of the metastable one. Here $\left\|\mathbf{M}-\frac{\mathbf{I}}{3}\right\|_{2}=|s| \sqrt{\frac{2}{3}}$. The stable and metastable states are drawn in solid curves. Nonzero values in $r_{1}$ and $M_{13}$ indicate the nonparallel nature of the states.

3.2. Concentrated limit. When $N>0$, the existence of the nonparallel steady states becomes complex when the parameters violate the conditions in the theorem. We look into this numerically. Our numerical results show that nonparallel 
steady states may exist; however, they are all unstable and there may exist up to two stable steady states exhibiting uniaxial symmetry.

Figure 3.2 depicts a representative solution diagram focusing on the stable steady states and on some nonparallel ones at $N=4.75$. Two stable uniaxial polar nematics may coexist in a finite range of $E$. One exists only at weak field strength while the other exists at all strengths. The short-lived stable state is metastable and its polarity vector is opposite to the direction of the imposed external field, in contrast to the globally stable state. In all the numerical experiments we have conducted, the nematic order of the stable state is always higher than the metastable state. Non-parallel steady states can exist in certain parameter ranges. However, they are unstable! $N=4.75$ is within the biphasic region in the case of pure nematics. At higher concentration, e.g., $N=6$, the scenario is essentially the same except for additional branches of unstable uniaxial branches that exist for a weak external field.

Figure 3.2 depicts the stable steady states along with some nonparallel unstable steady states at two selected values of $\alpha=2.5$ and $\alpha=3.5$. The metastable steady states become available at about $N>2.4$ at $\alpha=2.5, \chi_{0}=1, \mu=0.1, E=1$ while they exists for all $N>0$ at $\alpha=3.5, \chi_{0}=1, \mu=0.1, E=1$. The stronger dipole-dipole potential facilitates the metastable state. We note that the value of the order parameter in the globally stable steady state remains higher than that of the metastable state, indicating the slight loss of orientation order when the polarity vector is aligned against the direction of the external field.

\section{Conclusions}

We have extended the order reduction procedure developed in [20] to complete the study of steady states and their stability in extended nematics or nematic suspensions when the dipole-dipole and the excluded volume interaction is coupled with the external fields. The main results are summarized in the following.

- In the absence of permanent dipole or magnetic moment, both the polarity vector and the external field are shown to be aligned with one of the eigenvectors of the nematic order tensor. As a result of this, all equilibria can be characterized in terms of three scalar order parameters and a rotational group $S O(2)$ that leaves the external field invariant. Response phase diagrams of the order parameters with respect to the material parameters are obtained together with the stability of the steady states determined from the minimum of the free energy density. Globally stable steady states are uniaxial, prolate, purely nematic or polar nematics depending on the strength of the external field, the anisotropy, the strength of the dipole-dipole as well as the excluded volume interaction. A family of biaxial, metastable, steady states may exist with the major director aligned in the direction of the external field in the range of higher concentration. The polar nematics always exist in pairs with polar order $\pm s_{1}$.

- In the presence of the permanent dipole (or magnetic moment), the steady states are polar nematics. When the material parameters satisfy the conditions: $|\mu|>\chi_{0} E$ and $3 N \mu<\left|\alpha \chi_{0} E\right|$, the polarity vector and the external field are parallel to each other and are eigenvectors of the nematic order tensor; while the conditions are not satisfied, nonparallel steady solutions that are in plane with the polarity vector and the external field can exist, but are unstable. In any case, there may exist up to two stable steady states which are prolate, uniaxial, and polar. The globally stable steady state has its polarity vector parallel to the external field direction while the metastable state has 

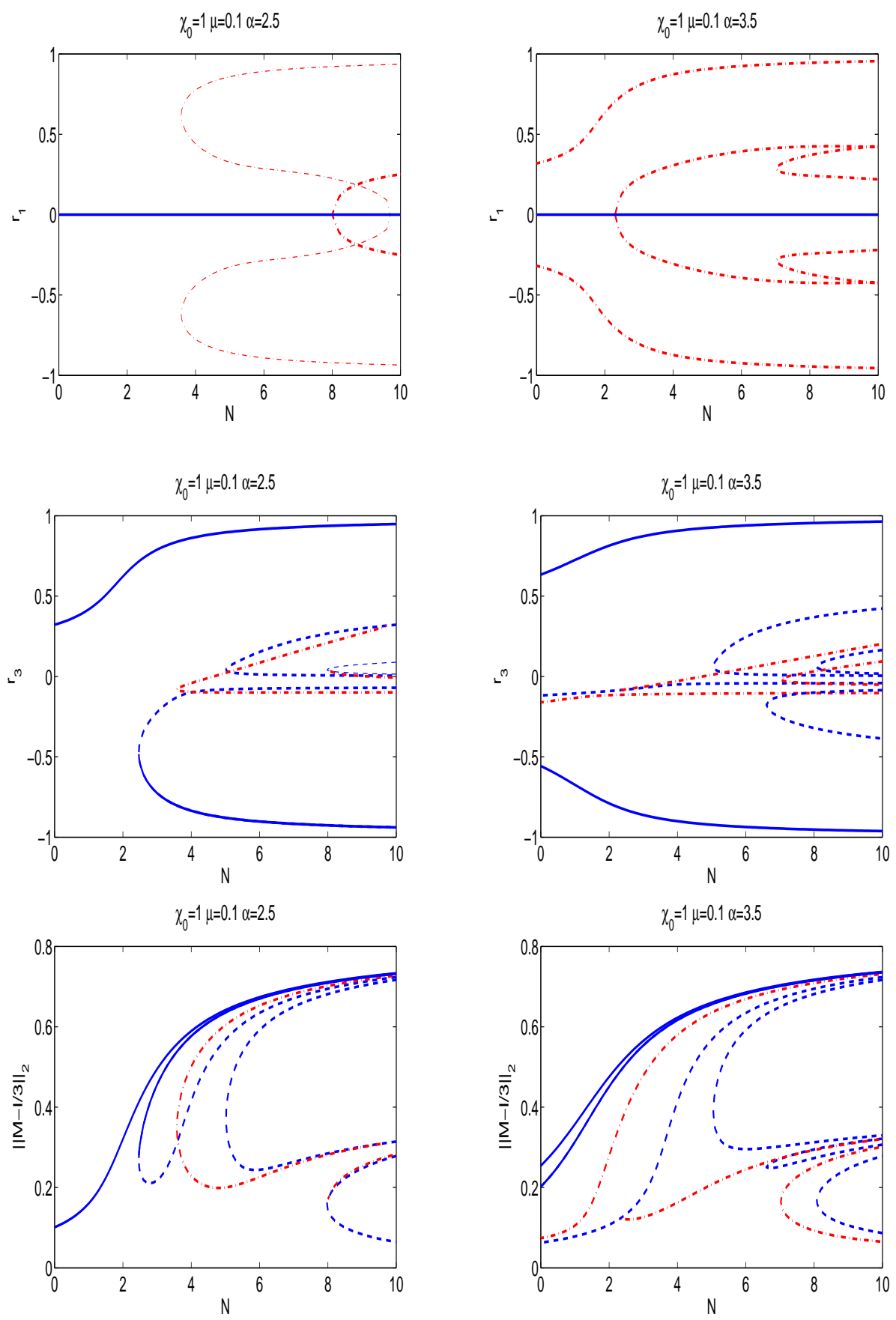

FIG. 3.3. The polarity vector components as functions of $N$ for $\alpha=2.5$ and $\alpha=3.5$, respectively. The other parameter values are $\chi_{0}=1, \mu=0.5, E=1$. The solid curves are stable states. The stable and metastable steady states are uniaxial, prolate, polar nematics with the polarity vector parallel to the external field. A metastable state can exist at higher concentration at $\alpha=2.5$ and for all $N$ at $\alpha=3.5$. The nonparallel steady states in which the polarity is not parallel to the external field can exist, but are unstable. The nematic order of the globally stable steady state is higher than that of the metastable one. 
its polarity vector opposite to the field. The former exists in any given parameter regimes while the latter only exists at regimes of high strength of the dipole-dipole interaction, high concentration, and strong external field. The presence of the permanent dipole breaks the symmetry in the total potential, yielding the two stable uniaxial polar nematics.

This study completes a series of studies on the potential for extended nematics and suspensions. The order reduction strategy used in these studies works for any total potential given as a finite sum of lower moments. It does not, however, apply to the Onsager potential, where the potential depends on infinitely many moments or spherical harmonics. New methods would have to be invented then.

\section{Appendix A. Free energy density and its second variation.}

A.1. Free energy density at steady states in order parameters. The free energy density at steady states in order parameters when the permanent dipole is absent is given by:

$$
\begin{aligned}
A[f] & =-k_{B} T \ln Z+\frac{k_{B} T}{2}\left(\alpha s_{1}^{2}+N\left(s^{2}-s \beta+\beta^{2}\right)\right), \\
Z & =\int_{\|m\|=1} e^{\alpha s_{1}+\frac{3 N}{2}\left[\left(s-\frac{\beta}{2}\right)\left(\cos ^{2} \theta-\frac{1}{3}\right)+\frac{\beta}{2} \sin ^{2} \theta \cos 2 \phi\right]} d m .
\end{aligned}
$$

Its first and second derivatives are given by

$$
\begin{aligned}
\frac{\partial A}{\partial s_{1}} & =\alpha k_{B} T\left(s_{1}-\langle\cos \theta\rangle\right), \\
\frac{\partial A}{\partial s} & =k_{B} T N\left(s+\frac{1-\beta}{2}-\frac{3}{2}\left\langle\cos ^{2} \theta\right\rangle\right), \\
\frac{\partial A}{\partial \beta} & =k_{B} T N\left(\beta+\frac{1-s}{2}-\frac{3}{2}\left\langle\sin ^{2} \theta \cos ^{2} \phi\right\rangle\right), \\
\frac{\partial^{2} A}{\partial s_{1}^{2}} & =\alpha k_{B} T-\alpha^{2} k_{B} T\left(\left\langle\cos ^{2} \theta\right\rangle-\langle\cos \theta\rangle^{2}\right) \\
& =\alpha k_{B} T-\alpha^{2} k_{B} T\left(\frac{1+2 s-\beta}{3}-s_{1}^{2}\right), \\
\frac{\partial^{2} A}{\partial s_{1} \partial s} & =-\frac{3 N \alpha k_{B} T}{2}\left(\left\langle\cos ^{3} \theta\right\rangle-\langle\cos \theta\rangle\left\langle\cos ^{2} \theta\right\rangle\right) \\
& =-\frac{3 N \alpha k_{B} T}{2}\left(\left\langle\cos ^{3} \theta\right\rangle-s_{1} \frac{1+2 s-\beta}{3}\right), \\
\frac{\partial^{2} A}{\partial s_{1} \partial \beta} & =-\frac{3 N \alpha k_{B} T}{2}\left(\left\langle\cos \theta \sin ^{2} \theta \cos ^{2} \phi\right\rangle-\langle\cos \theta\rangle\left\langle\sin ^{2} \theta \cos ^{2} \phi\right\rangle\right) \\
& =-\frac{3 N \alpha k_{B} T}{2}\left(\left\langle\cos \theta \sin ^{2} \theta \cos ^{2} \phi\right\rangle-s_{1} \frac{1-s+2 \beta}{3}\right), \\
\frac{\partial^{2} A}{\partial s^{2}} & =k_{B} T N-\frac{9 N^{2} k_{B} T}{4}\left(\left\langle\cos ^{4} \theta\right\rangle-\left\langle\cos ^{2} \theta\right\rangle^{2}\right) \\
& =k_{B} T N-\frac{9 N^{2} k_{B} T}{4}\left(\left\langle\cos ^{4} \theta\right\rangle-\frac{(1+2 s-\beta)^{2}}{9}\right),
\end{aligned}
$$




$$
\begin{aligned}
\frac{\partial^{2} A}{\partial s \partial \beta} & =-\frac{k_{B} T N}{2}-\frac{9 N^{2} k_{B} T}{4}\left(\left\langle\cos ^{2} \theta \sin ^{2} \theta \cos ^{2} \phi\right\rangle-\left\langle\cos ^{2} \theta\right\rangle\left\langle\sin ^{2} \theta \cos ^{2} \phi\right\rangle\right) \\
& =-\frac{k_{B} T N}{2}-\frac{9 N^{2} k_{B} T}{4}\left(\left\langle\cos ^{2} \theta \sin ^{2} \theta \cos ^{2} \phi\right\rangle-\frac{(1+2 s-\beta)(1-s+2 \beta)}{9}\right) \\
\frac{\partial^{2} A}{\partial \beta^{2}} & =k_{B} T N-\frac{9 N^{2} k_{B} T}{4}\left(\left\langle\sin ^{4} \theta \cos ^{4} \phi\right\rangle-\left\langle\sin ^{2} \theta \cos ^{2} \phi\right\rangle^{2}\right) \\
& =k_{B} T N-\frac{9 N^{2} k_{B} T}{4}\left(\left\langle\sin ^{4} \theta \cos ^{4} \phi\right\rangle-\frac{(1-s+2 \beta)^{2}}{9}\right) .
\end{aligned}
$$

At the isotropic branch $\left(s_{1}=0, s=0, \beta=0\right)$, the second variation of the free energy density is

$$
\begin{aligned}
\left.\delta^{2} A\right|_{s_{1}=0, s=0, \beta=0}= & k_{B} T\left[\left(\alpha-\alpha^{2} / 3\right) \delta^{2} s_{1}+N(1-N / 5) \delta^{2} s-N(1-N / 5) \delta s \delta \beta+\right. \\
& \left.N(1-N / 5) \delta^{2} \beta\right] .
\end{aligned}
$$

So, the isotropic equilibrium is unstable whenever $\alpha>3$ or $N>5$.

For the nonzero purely uniaxial nematic equilibria, $s \neq 0, \beta=0$,

$$
\delta^{2} A=k_{B} T \alpha\left(1-\frac{\alpha(1+2 s)}{3}\right) \delta s_{1}^{2}+k_{B} T N(\delta s, \delta \beta) \cdot C \cdot(\delta s, \delta \beta)^{T},
$$

where

$$
\begin{gathered}
C=\left(\begin{array}{cc}
\frac{5}{2}-\frac{3}{4 s}\left(\frac{e^{3 N s / 2}}{\psi(s)}-1\right)+\frac{N}{4}(1+2 s)^{2} & -\frac{5}{4}+\frac{3}{8 s}\left(\frac{e^{3 N s / 2}}{\psi(s)}-1\right)-\frac{N}{8}(1+2 s)^{2} \\
-\frac{5}{4}+\frac{3}{8 s}\left(\frac{e^{3 N s / 2}}{\psi(s)}-1\right)-\frac{N}{8}(1+2 s)^{2} & \frac{25}{16}-\frac{N}{32}\left(1-20 s-8 s^{2}\right)-\frac{9}{32 s}\left(\frac{e^{3 N s / 2}}{\psi(s)}-1\right)
\end{array}\right) \\
\psi(s)=\int_{0}^{1} e^{3 N s z^{2} / 2} d z
\end{gathered}
$$

A.2. Hessian of the free energy for dilute polar nematics. In the dilute limit, the excluded volume potential is neglected. The Hessian in the second variation of the free energy density in steady states in the order parameter space is given by

$$
\begin{aligned}
& \mathbf{H}=\alpha\left[\left(1-\alpha\left(\frac{1+2 s-\beta}{3}-s_{1}\right)\right) \mathbf{n n}+\left(1-\frac{\alpha}{3}(1-s+2 \beta)\right) \mathbf{n}_{\perp} \mathbf{n}_{\perp}+\right. \\
& \left.\left(1-\frac{\alpha}{3}(1-s-\beta)\right) \mathbf{n}^{*} \mathbf{n}^{*}\right]
\end{aligned}
$$

A.3. Hessian of the free energy density for the concentrated polar nematics. In this case, both the dipole-dipole and excluded volume interaction are included in the potential. We denote

$$
\begin{aligned}
\mathbf{M}= & \langle\mathbf{m m}\rangle \\
A_{12}= & -\frac{3 N \alpha}{2}\left[\langle\mathbf{m}\rangle\left(\left(M_{11}-M_{33}\right),\left(M_{22}-M_{33}\right), M_{12}, M_{13}, M_{23}\right)-\right. \\
& \left.\left\langle\left(\mathbf{m}\left(m_{1}^{2}-m_{3}^{2}\right), \mathbf{m}\left(m_{2}^{2}-m_{3}^{2}\right), \mathbf{m} m_{1} m_{2}, \mathbf{m} m_{1} m_{3}, \mathbf{m} m_{2} m_{3}\right)\right\rangle\right], \\
A_{22}= & \frac{(3 N)^{2}}{4}\left[\left\langle\left(m_{1}^{2}-m_{3}^{2}, m_{2}^{2}-m_{3}^{2}, m_{1} m_{2}, m_{1} m_{3}, m_{2} m_{3}\right)^{T}\right.\right. \\
& \left.\left(m_{1}^{2}-m_{3}^{2}, m_{2}^{2}-m_{3}^{2}, m_{1} m_{2}, m_{1} m_{3}, m_{2} m_{3}\right)\right\rangle- \\
& \left(M_{11}-M_{22}, M_{22}-M_{33}, M_{12}, M_{13}, M_{23}\right)^{T} \\
& \left.\left(M_{11}-M_{22}, M_{22}-M_{33}, M_{12}, M_{13}, M_{23}\right)\right] .
\end{aligned}
$$


Then, the Hessian in the second variation is given by

$$
\mathbf{H}=\left(\begin{array}{ll}
\alpha^{2}(\langle\mathbf{m m}\rangle-\langle\mathbf{m}\rangle\langle\mathbf{m}\rangle) & A_{12} \\
A_{12}^{T} & A_{22}
\end{array}\right) .
$$

A.4. Proof of the equivalence of the hydrodynamic equilibrium and the thermodynamic equilibrium in this model.

TheOREM A.1. The steady state solution of the Smoluchowski equation is given by the Euler-Lagrange equation of the free energy density.

Proof. The free energy density for homogeneous flows of rigid nematic polymers or rod/platelet dispersions is given by

$$
A[f]=k_{B} T \int_{\|\mathbf{m}\|=1}\left[f \ln f+\frac{V_{i}}{2} f+V_{e} f\right] d \mathbf{m},
$$

where $V_{i}$ and $V_{e}$ are the intermolecular potential and the external potential, respectively. The Euler-Lagrange equation obtained from minimizing the free energy density functional is

$$
\mu-\lambda=0
$$

where

$$
\mu=\frac{\delta A}{\delta f}=k_{B} T\left[\ln f+V_{i}+V_{e}\right]
$$

is the extended chemical potential and $\lambda$ is the Lagrange multiplier with respect to the constraint

$$
\int_{\|\mathbf{m}\|=1} f d \mathbf{m}=1 .
$$

$\mu=\lambda$ is the governing equation for the thermodynamic equilibria. Clearly it yields solutions of the Smoluchowski equation.

On the other hand, the steady state solution of the Smoluchowski equation is governed by

$$
\mathcal{R} \cdot(f \mathcal{R} \mu)=0 .
$$

We multiply the equation by $\mu$ and then integrate over the unit sphere $\|\mathbf{m}\|=1$ to yield

$$
\int_{\|\mathbf{m}\|=1} \mu \mathcal{R} \cdot f \mathbf{R} \mu d \mathbf{m}=-\int_{\|\mathbf{m}\|=1} f\|\mathcal{R} \mu\|^{2} d \mathbf{m}=0
$$

This implies

$$
f(\mathbf{m}, t) \mathcal{R} \mu=0 .
$$

Finally,

$$
\mathcal{R} \mu=0
$$


This is equivalent to $\mu=\lambda$.

Appendix B. The stability and the second variation of the free energy density. The stability of the steady states of the Smoluchowski equation in the Doi-Hess kinetic theory for extended nematics is studied by the second variation of the generalized free energy density. The free energy density for the material system subject to an external field is given by

$$
A[f]=k_{B} T\left[\int_{\|\mathbf{m}\|=1}\left(f \ln f+\frac{1}{2} V_{i}[f] f+V_{e} f\right) d \mathbf{m}\right] .
$$

We denote by $f_{e}$ the equilibrium pdf solution and $\delta f_{1}$ the perturbation of the equilibrium solution. Then, the second variation of the free energy density is given by

$$
\delta^{2} A\left[f_{e}\right]=\frac{\delta^{2}}{2} k_{B} T \int_{\|\mathbf{m}\|=1}\left(\frac{f_{1}^{2}}{f_{e}}+V_{i}\left[f_{1}\right] f_{1}\right) d \mathbf{m} .
$$

Let $f_{1}=f_{e} f_{2}$. The second variation reduces to

$$
\delta^{2} A=\frac{\delta^{2}}{2} k_{B} T\left\langle f_{2}^{2}+V_{i}\left[f_{e} f_{2}\right] f_{2}\right\rangle_{e},
$$

where $\langle(\bullet)\rangle_{e}$ denotes the ensemble average taken with respect to the equilibrium pdf. Recall that the equilibrium solution is in the Maxwell-Boltzmann form,

$$
f_{e}=\frac{1}{Z} e^{-V_{i}-V_{e}} .
$$

For the particular intermolecular potential studied in this paper, the intermolecular potential can be rewritten as

$$
V_{i}=-\alpha s_{1} m_{i}-\frac{3 N}{2}\left(s\left(m_{1}^{2}-1 / 3\right)+\beta\left(m_{2}^{2}-1 / 3\right)\right),
$$

where $\mathbf{M}=\mathbf{s}(\mathbf{n n}-\mathbf{I} / \mathbf{3})+\beta\left(\mathbf{n}_{\perp} \mathbf{n}_{\perp}-\mathbf{I} / \mathbf{3}\right)+\mathbf{I} / \mathbf{3}$ is a second moment tensor of the pdf and $i$ is an index between 1 and 3 . Without loss of generality, we set $i=1$ in this paper. We consider the perturbation

$$
\begin{gathered}
f_{2}=x-\langle x\rangle . \\
\int_{\|\mathbf{m}\|=1} f_{2} d \mathbf{m}=0 .
\end{gathered}
$$

First, we focus on a special perturbation that perturbs only the order parameters:

$$
x=\tilde{s}_{1} m_{1}+\left[\tilde{s}\left(m_{1}^{2}-1 / 3\right)+\tilde{\beta}\left(m_{2}^{2}-1 / 3\right)\right] .
$$

The equilibrium pdf is

$$
f=\frac{1}{Z} e^{\alpha s_{1} m_{1}+\frac{3 N}{2}\left[s\left(m_{1}^{2}-1 / 3\right)+\beta\left(m_{2}^{2}-1 / 3\right)\right]-V_{e}}
$$

Derive the Hessian for the quadratic term with respect to $\tilde{s}, \tilde{\beta}$. We note that

$$
s=\left\langle m_{1}^{2}-m_{3}^{2}\right\rangle, \beta=\left\langle m_{2}^{2}-m_{3}^{2}\right\rangle .
$$


We parameterize the molecular direction $\mathbf{m}$ in the frame of the eigenvectors of the second moment tensor

$$
\mathbf{m}=(\cos \theta, \sin \theta \cos \phi, \sin \theta \sin \phi) .
$$

In terms of the angles,

$$
s=\left\langle\cos ^{2} \theta-\sin ^{2} \theta \sin ^{2} \phi\right\rangle, \beta=\left\langle\sin ^{2} \theta \cos 2 \phi\right\rangle .
$$

The Hessian of the free energy density is given by

$$
\begin{aligned}
\frac{\partial^{2} A}{\partial \tilde{s}_{1}^{2}}= & 2\left[\left\langle m_{i}^{2}\right\rangle-\left\langle m_{i}\right\rangle^{2}-\left(\left\langle m_{i}^{2}\right\rangle^{2}+\frac{3 N}{2}\left\langle m_{i} \mathbf{m m}\right\rangle:\left\langle m_{i} \mathbf{m m}\right\rangle\right)\right], \\
\frac{\partial^{2} A}{\partial \tilde{s}_{1} \tilde{s}}= & 2\left\langle m_{i}\left(m_{1}^{2}-1 / 3\right)\right\rangle-2\left\langle m_{i}\right\rangle\left\langle m_{1}^{2}-1 / 3\right\rangle-\left(\alpha\left\langle m_{i}\left(m_{1}^{2}-1 / 3\right)\right\rangle\left\langle m_{i}^{2}\right\rangle+\right. \\
& \frac{3 N}{2}\left\langle\left(m_{1}^{2}-1 / 3\right) \mathbf{m m}\right\rangle:\left\langle m_{i} \mathbf{m m}\right\rangle+\left\langle m_{i}^{2}\right\rangle\left\langle m_{i}\left(m_{1}^{2}-1 / 3\right)\right\rangle+ \\
& \frac{3 N}{2}\left\langle m_{i} \mathbf{m m}\right\rangle:\left\langle\left(m_{1}^{2}-1 / 3\right) \mathbf{m m}\right\rangle, \\
\frac{\partial^{2} A}{\partial \tilde{s}_{1} \tilde{\beta}}= & 2\left\langle m_{i}\left(m_{2}^{2}-1 / 3\right)\right\rangle-2\left\langle m_{i}\right\rangle\left\langle m_{2}^{2}-1 / 3\right\rangle-\left(\alpha\left\langle m_{i}\left(m_{2}^{2}-1 / 3\right)\right\rangle\left\langle m_{i}^{2}\right\rangle+\right. \\
& \frac{3 N}{2}\left\langle\left(m_{2}^{2}-1 / 3\right) \mathbf{m m}\right\rangle:\left\langle m_{i} \mathbf{m m}\right\rangle+\left\langle m_{i}^{2}\right\rangle\left\langle m_{i}\left(m_{2}^{2}-1 / 3\right)\right\rangle+ \\
& \frac{3 N}{2}\left\langle m_{i} \mathbf{m m}\right\rangle:\left\langle\left(m_{2}^{2}-1 / 3\right) \mathbf{m m}\right\rangle, \\
\frac{\partial^{2} A}{\partial \tilde{s}^{2}}= & 2\left[\left\langle\left(m_{1}^{2}-1 / 3\right)^{2}\right\rangle-\left\langle\left(m_{1}^{2}-1 / 3\right)\right\rangle^{2}-\left(\alpha\left\langle m_{i}\left(m_{1}^{2}-1 / 3\right)\right\rangle^{2}+\right.\right. \\
& \left.\left.\frac{3 N}{2}\left\langle\left(m_{1}^{2}-1 / 3\right) \mathbf{m m}\right\rangle:\left\langle\left(m_{1}^{2}-1 / 3\right) \mathbf{m m}\right\rangle\right)\right], \\
\frac{\partial^{2} A}{\partial \tilde{s} \tilde{\beta}}= & 2\left\langle\left(m_{1}^{2}-1 / 3\right)\left(m_{2}^{2}-1 / 3\right)\right\rangle-2\left\langle\left(m_{1}^{2}-1 / 3\right)\right\rangle\left\langle\left(m_{2}^{2}-1 / 3\right)\right\rangle- \\
& \left(2 \alpha\left\langle m_{i}\left(m_{1}^{2}-1 / 3\right)\right\rangle\left\langle m_{i}\left(m_{2}^{2}-1 / 3\right)\right\rangle+3 N\left\langle\left(m_{2}^{2}-1 / 3\right) \mathbf{m m}\right\rangle:\left\langle\left(m_{1}^{2}-1 / 3\right) \mathbf{m m}\right\rangle\right), \\
\frac{\partial^{2} A}{\partial \tilde{\beta}^{2}}= & 2\left[\left\langle\left(m_{2}^{2}-1 / 3\right)^{2}\right\rangle-\left\langle\left(m_{2}^{2}-1 / 3\right)\right\rangle^{2}-\left(\alpha\left\langle m_{i}\left(m_{2}^{2}-1 / 3\right)\right\rangle^{2}+\right.\right. \\
& \left.\left.\frac{3 N}{2}\left\langle\left(m_{2}^{2}-1 / 3\right) \mathbf{m m}\right\rangle:\left\langle\left(m_{2}^{2}-1 / 3\right) \mathbf{m m}\right\rangle\right)\right] .
\end{aligned}
$$

Here we normalize the free energy density by $k_{B} T$ and $i=1$.

\section{REFERENCES}

[1] A.S. Bhandar and J.M. Wiest, Mesoscale constitutive modeling of magnetic dispersions, Journal of Colloid and Interface Science, 257, 371-382, 2003.

[2] B. Bird, R.C. Armstrong and O. Hassager, Dynamics of Polymeric Liquids, Vol 1 and 2, John Wiley and Sons, New York, 1987.

[3] F. Biscarini, C. Chiccoli, P. Pasini and C. Zannoni, On a simple model of ferroelectricity in liquid crystals, Journal of Non-Crystalline Solids, 131-133, 1190-1193, 1991.

[4] P.M. Chaikin and T.C. Lubensky, Principles of Condensed Matter Physics, Cambridge University Press, Cambridge, 1995.

[5] P. Constantin, I. Kevrekidis and E.S. Titi, Asymptotic states of a Smoluchowski equation, Arch. Rat. Mech. Anal., 174, 365-384, 2004. 
[6] P. Constantin, I. Kevrekidis and E.S. Titi, Remarks on a Smoluchowski equation, Discrete and Continuous Dynamical Systems, 11, 101-112, 2004.

[7] P. Constantin and J. Vukadinovic, Note on the number of steady states for a $2 D$ Smoluchowski equation, Nonlinearity, 18, 441-443, 2005.

[8] P.G. de Gennes and J. Prost, The Physics of Liquid Crystals, 2nd ed., Oxford University Press, New York, 1993.

[9] M. Doi and S.F. Edwards, The Theory of Polymer Dynamics, Clarendon Press, Oxford, 1986.

[10] I. Fatkullin and V. Slastikov, Critical points of the Onsager functional on a sphere, Nonlinearity, 18, 2565-2580, 2005.

[11] M.G. Forest and Q. Wang, Monodomain response of finite-aspect-ratio macromolecules in shear and related linear flows, Rheological Acta, 42, 20-46, 2005.

[12] M.G. Forest, Q. Wang and R. Zhou, Symmetries of the Doi kinetic theory for nematic polymers of finite and infinite aspect ratio: at rest and in linear flows, Physical Review E, 66(3), P031712, 2003.

[13] M.G. Forest, Q. Wang and R. Zhou, Full tensor alignment criteria for sheared nematic polymers, J. of Rheology, 47(1), 105-127, 2003.

[14] M.G. Forest, Q. Wang and R. Zhou, The weak shear phase diagram for nematic polymers, Rheological Acta, 43(1), 17-37, 2004.

[15] M.G. Forest, Q. Wang and R. Zhou, The flow-phase diagram of Doi-Hess theory for sheared nematic polymers II: finite shear rates, Rheological Acta, 44(1), 80-93, 2004

[16] M.G. Forest, R. Zhou and Q. Wang, Scaling behavior of kinetic orientational distributions for dilute nematic polymers in weak shear, J. Non-Newtonian Fluid Mechanics, 116, 183-204, 2004.

[17] M.G. Forest, R. Zhou and Q. Wang, Chaotic boundaries of nematic polymers in mixed shear and extensional flows, Physical Review Letters, 93(8), 088301-088305, 2004.

[18] A. Gopinath, L. Mahadevan and R.C. Armstrong, Transitions to nematic states in homogeneous suspensions of high aspect ratio magnetic rods, Phys. Fluids, 18, 028102-028106, 2006.

[19] S. Hess, Fokker-Planck-equation approach to flow alignment in liquid crystals, Z Naturforschung 31a, 1034-1037, 1976.

[20] G. Ji, Q. Wang, P. Zhang and H. Zhou, Study of phase transition in homogeneous, rigid extended nematics and magnetic suspensions using an order-reduction method, Physics of Fluids, 18, 123103, 2006.

[21] T.J. Krieger and H.M. James, Successive orientation transitions in crystals, Journal of Chem. Phys., 22(5), 796-814, 1954.

[22] R.G. Larson, Arrested tumbling in shearing flows of liquid crystal polymers, Macromolecules, 23, 3983-3992, 1990.

[23] R.G. Larson and H. Ottinger, Effects of molecular elasticity on out-of-plane orientations in shearing flows of liquid-crystalline polymers, Macromolecules 24, 6270-6282, 1991.

[24] R.G. Larson, The Rheology of Complex Fluids, Oxford University Press, New York, 1998.

[25] T. Li and P. Zhang, Mathematical analysis of multi-scale models of complex fluids, Commun. Math. Sci., 5(1), 1-51, 2007.

[26] B. Jourdain, T. Lelievre and C. LeBris, Existence of solution for a micro-macro model of polymeric fluid: the FENE model, Journal of Functional Analysis, 209, 162-193, 2004.

[27] K.M. Leung and L. Lei, Phase transitions of bowlic liquid crystals, Mol. Cryst. Liq. Cryst., 146, 71-76, 1987.

[28] L. Lei, Bowlic liquid crystals, Mol. Cryst. Liq. Cryst., 146, 41-45, 1987.

[29] W. Maier and A. Saupe, A simple molecular-statistics theory of the liquid-crystalline state, Z. Naturforsch., 13a, 564-566, 1958.

[30] W. Maier and A. Saupe, A simple molecular-statistics theory of the liquid-crystalline phase. Part I, Z. Naturforsch., 14a, 882-889, 1959.

[31] W. Maier and A. Saupe, A simple molecular-statistics theory of the liquid-crystalline phase. Part II, Z. Naturforsch., 15a, 287-292, 1960.

[32] G.D. Matteis and E.G. Virga, Tricritical points in biaxial liquid crystal phases, Phys. Rev. E, 71, 061703, 2005.

[33] S. Ramalingam and R.C. Armstrong, Analysis of isothermal spinning of liquid crystalline polymers, J. Rheology, 37(6), 1141-1169, 1993.

[34] C. Luo, H. Zhang and P. Zhang, The structure of equilibrium solutions of the one-dimensional Doi equation, Nonlinearity, 18, 379-389, 2005.

[35] H. Liu, H. Zhang and P. Zhang, Axial symmetry and classification of stationary solutions of Doi-Onsager equation on the sphere with Maier-Saupe potential, Commun. Math. Sci., 3, 201-218, 2005. 
[36] Q. Wang, Comparative studies on closure approximations in flows of liquid crystal polymers. I. Elongational flows, Journal of Non-Newtonian Fluid Mechanics, 72, 141-162, 1997.

[37] Q. Wang, Comparative studies on closure approximations in flows of liquid crystal polymers. II. Fiber flows, Journal of Non-Newtonian Fluid Mechanics, 72, 163-185, 1997.

[38] Q. Wang, S. Sircar and H. Zhou, Steady state solutions of the Smoluchowski equation for nematic polymers under imposed fields, Commun. Math. Sci., 4(3), 605-620, 2005.

[39] D. Wei and G. N. Patey, Orientational order in simple dipolar liquids: computer simulation of ferroelectric nematic phase, Phys. Rev. Lett., 68(13), 2043-2045, 1992.

[40] H. Zhou, H. Wang, M.G. Forest and Q. Wang, A new proof on axisymmetric equilibria of a three-dimensional Smoluchowski equation, Nonlinearity, 18, 2815-2825, 2005.

[41] H. Zhou, H. Wang, Q. Wang and M.G. Forest, Characterization of stable kinetic equilibria of rigid, dipolar rod ensembles for coupled dipole-dipole and Maier-Saupe potentials, Nonlinearity, 20, 277-297, 2007.

[42] H. Zhou, H. Wang and Q. Wang, Nonparallel solutions of extended nematic polymers under an external field, Discrete and Continuous Dynamical Systems-Series B, 7(4), 907-929, 2007. 